\title{
Perturbative expansions in QCD and analytic properties of $\alpha_{s}$
}

\author{
Yu.A.Simonov \\ State Research Center, \\ Institute of Theoretical and Experimental Physics, Moscow, Russia
}

\begin{abstract}
It is shown that analytic properties of standard QCD perturbation theory contradict known spectral properties,and contain in particular IR generated ghost poles and cuts. As an outcome the rigorous background perturbation theory is developed and its analytic properties are shown to be in agreement with general requirements. In a limiting case of large $N_{c}$, when QCD amplitudes contain only pole singularities, the strong coupling constant $\alpha_{s}(Q)$ is shown to be also meromorfic function of external momenta.

Some simple models and examples are given when nonperturbative $\beta$ function and $\alpha_{s}(Q)$ can be written explicitly. General form of amplitudes at large $N_{c}$ is given in the framework of background perturbation theory and its correspondence with standard perturbation theory at large momenta is demonstrated in the example of $e^{+} e^{-}$annihilation. For time-like momenta the background coupling constant differs drastically from the standard one but the background series averaged over energy intervals has the same (AF) asymptotics at large momenta as in the standard perturbation theory.
\end{abstract}

\section{Introduction}

The standard perturbation theory (SPT) in QCD is well developed both on the theoretical and on phenomenological levels [1], 2] , and constitutes the major and best understood part of QCD. Successful applications of perturbative expansions for processes with large momenta are numerous and impressive. 
Howerever there are several basic difficulties in SPT (it is assumed that RG improvements such as partial summation of large logarithms are automatically included in SPT), namely

1) Analytic properties of SPT amplitudes do not correspond to the expected spectral behaviour. In particular, there appear ghost poles and cuts even in the Euclidean region of momenta, where one expects amplitudes to be holomorphic.

These ghost singularities are due to analytic properties of $\mathrm{RG}$ improved $\alpha_{s}(Q)$ and are connected with IR divergence of $\alpha_{s}(Q)$. Also in the Minkowskian region of momenta $\alpha_{s}(Q)$ have logarithmic cuts which the physical amplitude should not possess, and they are therefore artefact of SPT.

In short, amplitudes computed in SPT have analytic behaviour which has nothing to do with physical thresholds and cuts due to creation of hadrons. Therefore one may only speak about some duality relations between SPT amplitudes and physical amplitudes integrated over sufficiently large energy interval.

2) Another basic defect of SPT is the lack of convergence of standard perturbative series. There are arguments that the latter is an asymptotic series [3], and in all cases it is not clear where the series should be cut off. (Some hints that the three-loop contribution deteriorates the physical results in the $1-1.5 \mathrm{GeV}$ region are contained in [3] and [4]). Moreover, the Landau ghost poles give rise to the appearence of the so-called IR renormalons [5], which make the sum of perturbative series undefined even in the Borel sense.

The attempts to connect the IR renormalons with the nonperturbative contributions may have only qualitative character at best. Therefore strictly speaking the notion of the sum of SPT series has no definite meaning, and one may only hope that at large Euclidean momenta the first few terms of RG improved perturbative series describe the asymptotics of physical amplitudes with reasonable accuracy.

To improve the situation several approaches have been developed. First of all, it was understood long ago, that in addition to SPT also nonperturbative contributions should be taken into account. Technically the latter have been introduced as the local terms (condensates) in the OPE, and on this foundation the QCD sum rules have been introduced [6]. In this way one can approach the low-energy region around 1-2 GeV and a lot of useful physical information have been obtained in this method for the last 20 years [7].

However in this method one does not solve both problems of SPT described above, and instead postulates that in addition to a few first SPT 
terms one can add few power terms to imitate behaviour of physical amplitude not only in asymptotic region but also in the region of few $\mathrm{GeV}$. In the lower energy region the OPE series has at least double divergence: due to explosion of $\alpha_{s}$ near the Landau ghost pole and due to explosion of power terms $\frac{C_{n}}{Q^{2 n}}$.

It is important to note that the original OPE and QCD sum rules [6] have been properly defined only in the Euclidean region, and the transition to the time-like region is assumed to be done aposteriori, after all calculations are done in the Eulidean region.

Definition of $\alpha_{s}\left(Q^{2}\right)$ in the Minkowskian region of $Q^{2}, Q^{2}<0$, is a problem by itself in SPT, since formal analytic continuation of two - and more-loop expressions for $\alpha_{s}\left(Q^{2}\right)$ yield complex expressions violating explicit unitarity conditions in nonasymptotic region.

Moreover as was stressed in [8], the invariant coupling $\bar{g}(Q)$ can be defined only in the space-like domain, and "inside the RG formalism there is no simple means for defining $\bar{g}(Q)$ in the time-like region".

New formalism for definition of $\alpha_{s}\left(Q^{2}\right)$ both in space-like and time-like region was developed in 9] and a new method was suggested, called analytic perturbation theory (see [8] for a review and further references), where $\alpha_{s}\left(Q^{2}\right)$ is forced to be analytic for $Q^{2}>0$ and a special procedure is envisaged to continue $\alpha_{s}$ analytically into the time-like region. The method allows to get rid of the Landau ghost pole and cuts and seriously improves convergence of the perturbative series.

It is clear however that this is not unique way of analytic definition in the whole complex plane of $z$.

In what follows we shall choose a completely different strategy. To simplify matter we shall consider below the limiting case of large $N_{c}$. In this case one can be sure that all physical amplitudes contain only poles as functions of external momenta [10, and we shall require that perturbative expansion would reproduce the meromorphic properties of physical amplitudes, i.e. $\alpha_{s}\left(Q^{2}\right)$ have only singularities in the time-like region, and those are poles.

To make this goal, one needs to use Background Perturbation Theory (BPT) instead of the standard one, and we shall derive rigorous formalism based on BPT developed in the seventies and eighties [11] and generalized in [12]-14 to include nonclassical background and averaging over background fields.

In this way one obtains a systematic formalism which allows to express 
all terms of BPT through the irreducible correlators of background fields (integrals thereof) and renormalized coupling constant $\alpha_{B}$, which we shall denote $\alpha_{B}(s)$ to distinguish it from $\alpha_{s}(s)$ in SPT.

It was argued in 14 that $\alpha_{B}(s)$ and physical amplitudes satisfies the same RG equations, in particular Ovsyannikov-Callan-Simanzyk (OCS) equations, and the important difference in $\alpha_{B}\left(Q^{2}\right)$ from $\alpha_{s}\left(Q^{2}\right)$ lies in the character of its dependence on $Q^{2}$. It was demonstrated in [12]-14] that $\alpha_{B}\left(Q^{2}\right)$ has the property of freezing, or saturation at small $Q^{2}$, i.e. it tends to a finite limit $\alpha_{B}(0)$ when $Q^{2} \rightarrow 0$ and has no singularities in the whole Euclidean region $Q^{2} \geq 0$.

This behaviour of $\alpha_{B}\left(Q^{2}\right)$ was tested repeatedly in $e^{+} e^{-}$annihilation [13, 14], in fine structure of charmonium [15] and bottomonioum levels [16] and recently [4] in comparison with accurate lattice data on small-distance behaviour of $\alpha_{L}(R)$. In all cases the same form of solution for $\alpha_{B}$ was used without free parameters which produced results in a good agreement with experimental and lattice data. In this way the phenomenon of freezing (saturation) was demonstrated both theoretically as a result of confinement in background fields [12], and phenomenologically in comparison with lattice and experimental data.

With all that some important theoretical questions were not answered, first of all what happens with $\alpha_{B}(s)$ in the Minkowskian (time-like) region and what are analytic properties of $\alpha_{B}(s)$ in the whole $s$-plane.

Secondly, what is connection of $\alpha_{B}(s)$ and other nonperturbative definitions of $\alpha(s)$, e.g. lattice definitions of $\alpha-\alpha_{L}(s)$.

In the present paper it is our purpose to study the problem of BPT in the large $N_{c}$ limit, trying to elucidate several aspects.

Firstly, we formulate the foundations of BPT and rules for calculation of perturbative series, starting from the purely nonperturbative term.

Secondly, we derive the RG equations taking into account that nonperturbative background is in general not classical solution and subject to the vacuum averaging, which due to the 'tHooft identity can be done independently of the perturbative field averaging.

Thirdly, we find the most general solution of RG equations and in particular the nonperturbative $\beta$ - function which has known lowest term expansion, at the same time $\alpha_{B}\left(Q^{2}\right)$ is represented as a sum of pole terms.

To understand which kind of singularities $\alpha_{B}\left(Q^{2}\right)$ may have compatible with analytic properties of physical amplitudes we consider a simplified model and demonstrate that any finite order perturbative expansion has ad- 
ditional singularities which are eliminated when a partial summation of the perturbative series is done.

As a next step we formulate the generic perturbative expansion for a physical amplitude, choosing an example of $e^{+} e^{-}$annihilation into hadrons and write it as a sum over poles in the time-like region with calculable coefficients.

At this point one may wonder how this meromorphic expansion is connected to SPT expansion, where the perturbative series is in powers of $\alpha_{s}\left(Q^{2}\right)$, which contain logarithmic functions of $Q^{2}$ and not meromorphic. The answer to this connection was given in the analysis of the lowest term of $e^{+} e^{-}$annihilation - the hadronic part of the photon self-energy. The latter is a sum over meson poles at large $N_{c}$ and has a proper logarithmic behaviour $\log Q^{2}$ at large $Q^{2}$. We demonstrate that a similar correspondence takes place also for higher terms of BPT, and formulate conditions on the coefficients of meromorphic expansion which are necessary to reproduce the known SPT expansion at large $Q^{2}$.

Discussion of practical applications and comparison to other approaches concludes the paper.

The exact structure of paper is as follows. In section 2 general rules of BPT are given, and Green's functions for valence quarks, gluons and hadrons are written explicitly.

In section 3 the $\mathrm{RG}$ equations are written down in the case of most general background and the point of RG scheme is discussed and a particular solution of RG equations for $\beta$ - function and $\alpha_{B}\left(Q^{2}\right)$ is presented.

In section 4 a simple model is discussed where some BPT subseries can be summed up explicitly.

In section 5 nonasymptotic terms in the spectral sums are calculated and in section 6 behaviour of perturbtive series in the region of time-like $Q^{2}$ is studied in detail.

Discussion and outlook is contained in the concluding section.

\section{Background Perturbation Theory for arbi- trary background}

Gluon field plays in QCD two different roles:

i) gluons are propagating, and at small distances this process can be described perturbatively, leading in particular to color Coulomb interaction 
between quarks (antiquarks);

ii) gluons form a kind of condensate, which serves as a background for the propagating perturbative gluons and quarks. This background is Euclidean and ensures phenomena of confinement and CSB.

Correspondingly we shall separate the total gluonic field $A_{\mu}$ into perturbative part $a_{\mu}$ and nonperturbative (NP) background $B_{\mu}$ :

$$
A_{\mu}=B_{\mu}+a_{\mu}
$$

There are many questions about this separation, which may be answered now only partially, e.g. what exactly is the criterion of separation. Possible answer is that perturbative fields $a_{\mu}$ get their dimension from distance (momentum), and therefore all correlators of fields $a_{\mu}$ (in absence of $B_{\mu}$ ) are singular and made of inverse powers of $(x-y)$ and logarithms, where enters the only dimensional parameter of perturbative QCD $-\Lambda_{Q C D}$. Therefore evidently any dimensionful constant, like hadronic masses or string tension cannot be obtained as a perturbation series. In contrast to that, NP fields $B_{\mu}$ have mass dimension due to the violation of scale invariance which is intrinsically present in the gluodynamics Lagrangian. The origin of separation (11) is clearly seen in the solutions of nonlinear equations for field correlators [17]: a perturbative solution of those leads to singular power-like field correlator, whereas at large distances there is a selfconsistent solution of the equations, decaying exponentially with distance with arbitrary mass scale, since equations in [17] are scale-invariant. Full solution including intermediate distances produces mixed perturbative-nonperturbative terms, containing both inverse powers of distance and exponentials. For these terms criterion of separation fails.

One can avoid formally the question of separation principle (and of double counting) using t'Hooft identity [13], which allows to integrate in (1) independently over $B_{\mu}$ and $a_{\mu}$ :

$$
Z=\frac{1}{N^{\prime}} \int D B_{\mu} \eta(B) D \psi D \bar{\psi} D a_{\mu} e^{L_{t o t}}
$$

Here the weight $\eta(B)$ is arbitrary and may be taken as constant.

To define the perturbation theory series in $g a_{\mu}$ one starts from (四) and rewrites the Lagrangian as follows:

$$
L_{t o t}=L_{g f}+L_{g h}+L(B+a)=
$$




$$
L_{0}+L_{1}+L_{2}+L_{i n t}+L_{g f}+L_{g h}
$$

where $L_{i}$ have the form:

$$
\begin{gathered}
L_{2}(a)=\frac{1}{2} a_{\nu}\left(\hat{D}_{\lambda}^{2} \delta_{\mu \nu}-\hat{D}_{\mu} \hat{D}_{\nu}+i g \hat{F}_{\mu \nu}\right) a_{\mu}= \\
=\frac{1}{2} a_{\nu}^{c}\left[D_{\lambda}^{c a} D_{\lambda}^{a d} \delta_{\mu \nu}-D_{\mu}^{c a} D_{\nu}^{a d}-g f^{c a d} F_{\mu \nu}^{a}\right] a_{\mu}^{d}, \\
D_{\lambda}^{c a}=\partial_{\lambda} \cdot \delta_{c a}+g f^{c b a} B_{\lambda}^{b} \equiv \hat{D}_{\lambda}, \quad F_{\mu \nu}^{a}=\partial_{\mu} B_{\nu}^{a}-\partial_{\nu} B_{\mu}^{a}+g f^{a b c} B_{\mu}^{b} B_{\nu}^{c} \\
L_{0}=-\frac{1}{4}\left(F_{\mu \nu}^{a}(B)\right)^{2} ; \quad L_{1}=a_{\nu}^{c} D_{\mu}^{c a}(B) F_{\mu \nu}^{a} \\
L_{\text {int }}=-\frac{1}{2}\left(D_{\mu}(B) a_{\nu}-D_{\nu}(B) a_{\mu}\right)^{a} g f^{a b c} a_{\mu}^{b} a_{\nu}^{c}-\frac{1}{4} g^{2} f^{a b c} a_{\mu}^{b} a_{\nu}^{c} f^{a e f} a_{\mu}^{e} a_{\nu}^{f} \\
L_{\text {ghost }}=-\theta_{a}^{+}\left(D_{\mu}(B) D_{\mu}(B+a)\right)_{a b} \theta_{b} .
\end{gathered}
$$

It is convenient to prescribe to $B_{\mu}, a_{\mu}$ the following gauge transformations

$$
\begin{gathered}
a_{\mu} \rightarrow U^{+} a_{\mu} U \\
B_{\mu} \rightarrow U\left(B_{\mu}+\frac{i}{g} \partial_{\mu}\right) U
\end{gathered}
$$

and to impose on $a_{\mu}$ the background gauge condition

$$
G^{a} \equiv\left(D_{\mu} a_{\mu}\right)^{a}=\partial_{\mu} a_{\mu}^{a}+g f^{a b c} B_{\mu}^{b} a_{\mu}^{c}=0
$$

In this case the ghost field have to be introduced as in (6) and the gaugefixing term is $L_{g f}=-\frac{1}{2 \xi}\left(G^{a}\right)^{2}$ One can write the resulting partition function as

$$
Z=\frac{1}{N^{\prime}} \int D B \eta(B) Z(J, B)
$$

where

$$
Z(J, B)=\int D \psi D \bar{\psi} D a_{\mu} D \theta D \theta^{+} e^{L_{t o t}+\int J_{\mu} a_{\mu} d x}
$$

We now can identify the propagator of $a_{\mu}$ from the quadratic terms in Lagrangian $L_{2}(a)-\frac{1}{2 \xi}\left(G^{a}\right)^{2}$

$$
G_{\nu \mu}^{a b}=\left[\hat{D}_{\lambda}^{2} \delta_{\mu \nu}-\hat{D}_{\mu} \hat{D}_{\nu}+i g \hat{F}_{\mu \nu}+\frac{1}{\xi} \hat{D}_{\nu} \hat{D}_{\mu}\right]_{a b}^{-1}
$$


It will be convenient sometimes to choose $\xi=1$ and end up with the wellknown form of propagator in - what one would call - the background Feynman gauge

$$
G_{\nu \mu}^{a b}=\left(\hat{D}_{\lambda}^{2} \cdot \delta_{\mu \nu}-2 i g \hat{F}_{\mu \nu}\right)^{-1}
$$

Integration over ghost and gluon degrees of freedom in (11) yields

$$
\begin{array}{r}
Z(J, B)=\operatorname{const}(\operatorname{det} W(B))_{r e g}^{-1 / 2}\left[\operatorname{det}\left(-D_{\mu}(B) D_{\mu}(B+a)\right]_{a=\frac{\delta}{\delta J}} \times\right. \\
\times\left.\left\{1+\sum_{l=1}^{\infty} \frac{S_{i n t}}{l !}\left(a=\frac{\delta}{\delta J}\right)\right\} \exp \left(-\frac{1}{2} J W^{-1} J\right)\right|_{J_{\nu}=D_{\mu}(B) F_{\mu \nu}(B)}
\end{array}
$$

where $W=G^{-1}$, and $G$ is defined in (12-13).

Let us mention the important property of the background Lagrangian (3): under gauge tranformations the fields $a_{\mu}, B_{\mu}$ transform as in (7-8) and all terms of (3), including the gauge fixing one $\frac{1}{2}\left(G^{a}\right)^{2}$ are gauge invariant. That was actually one of the aims put forward by G. 'tHooft in [1], and it has important consequences too: (i) any amplitude in perturbative expansion in $g a_{\mu}$ of (11) and (14) corresponding to a generalized Feynman diagram, is separately gauge invariant (for colorless initial and final states of course).

(ii) Due to gauge invariance of all terms, the renormalization is specifically simple in the background field formalism [11], since the counterterms enter only in gauge-invariant combination, e.g. $F_{\mu \nu}^{2}$ and hence $Z$-factors $Z_{g}$ and $Z_{A}$ are connected. We shall exploit this fact in Sections 6 and 7.

Let us turn now to the term $L_{1}$ in (3) $L_{1}=2 \operatorname{tr}\left(a_{\nu} D_{\mu} F_{\mu \nu}\right)$, which is usually missing in the standard background field formalism [11], since one tacitly assumes there that the background $B_{\mu}$ is a classical solution,

$$
D_{\mu}(B) F_{\mu \nu}(B)=0
$$

Here we do not impose the condition (15) and consider any background, classical or purely quantum fluctuations. Let us estimate the influence of the vertex $L_{1}$. In general it leads to the shift of the current $J_{\mu}$ in the expression for the perturbative series (14). Physically it means that at each point the background $B_{\mu}$ can generate a perturbative gluon via the vertex $\left(a_{\mu} D_{\nu} F_{\nu \mu}\right)$, and this vertex is proportional to the degree of "nonclassicality" of $B_{\mu}$. For the quasiclassical vacuum, like the instanton model, the average value $<\left(D_{\mu} F_{\mu \nu}\right)^{2}>$ over instanton ensemble is less then $0\left(\rho^{4} / R^{4}\right)$ and is small ( 
at most of the order of few percents) while average of $\left\langle D_{\mu} F_{\mu \nu}\right\rangle$ vanishes in the symmetric vacuum. All this is true provided the instanton gas stabilizes at small density.

Let us estimate the effect of $L_{1}$ in the general quantum case. To this end we calculate as in [12] the contribution of $L_{1}$ to the gluon propagator.

If one denotes by $<>_{a}$ the integral $D a_{\mu}$ with the weight $L_{t o t}$ as in (11), we obtain

$$
\begin{array}{r}
<a_{\mu_{1}}\left(x_{1}\right) a_{\mu_{2}}\left(x_{2}\right)>_{a}=G_{\mu_{1} \mu_{2}}\left(x_{1}, x_{2}\right)+\Delta_{\mu_{1} \mu_{2}}^{B} \\
\Delta_{\mu_{1} \mu_{2}}^{B} \equiv \int d^{4} y_{1} d^{4} y_{2} G_{\mu_{1} \nu_{1}}\left(x_{1}, y_{1}\right) D_{\rho} F_{\rho \nu_{1}}\left(y_{1}\right) D_{\lambda} F_{\lambda \nu_{2}}\left(y_{2}\right) G_{\nu_{2} \mu_{2}}\left(y_{2}, x_{2}\right)
\end{array}
$$

The gluon Green's function $G_{\mu \nu}$ is given in (13) and depends on the background field $B_{\mu}$, as well as $D_{\mu}$ and $F_{\rho \lambda}$. To get a simple estimate of $\Delta$ we replace $G_{\mu \nu}$ by the free Green's function $G_{\mu \nu}^{(0)}$ and take into account that

$$
<D_{\rho} F_{\rho \nu_{1}}\left(y_{1}\right) D_{\lambda} F_{\lambda \nu_{2}}\left(y_{2}\right)>_{B} \Rightarrow \frac{\partial}{\partial y_{1} \rho} \frac{\partial}{\partial y_{2 \lambda}}<F_{\rho \nu_{1}}\left(y_{1}\right) F_{\lambda \nu_{2}}\left(y_{2}\right)>
$$

and for the latter we use the representation [18] in terms of two independent Lorentz structures, $D\left(y_{1}-y_{2}\right)$ and $D_{1}\left(y_{1}-y_{2}\right)$. The contribution of $D$ (that of $D_{1}$ is of similar character) in the momentum space is

$$
\Delta_{\mu_{1} \mu_{2}}^{B}(k) \sim \frac{\left(k^{2} \delta_{\mu_{1} \mu_{2}}-k_{\mu_{1}} k_{\mu_{2}}\right)}{k^{4}} D(k)
$$

where

$$
D(k)=\int d^{4} y e^{i k y} D(y)
$$

Insertion in (19) of the exponential fall-off for $D(y)$ found in lattice calculations [19] yields

$$
D(k)=\frac{<F^{2}(0)>}{\left(N_{c}^{2}-1\right)} \cdot \frac{\pi^{2} \mu}{\left(\mu^{2}+k^{2}\right)^{5 / 2}} .
$$

With $\mu \approx 1 G e V, \quad D(k) \approx 0.12$. Thus $\Delta^{B}(k)$ is a soft correction fast decreasing with $k$ as $k^{-5}$ to the perturbative gluon propagator. 


\section{Renormalization group improvement of per- turbative series and analytic properties of $\alpha_{s}\left(Q^{2}\right)$}

The most important property of the Background method discussed in the previous chapter, is the gauge invariance of the total Lagrangian including the gauge-fixing term $L_{g f}$.

As a consequence of this the counterterms in the renormalization procedure have to be gauge invariant too and this fact establishes connection between $Z$-factors of charge and background field [11], namely, if

$$
g_{0}=Z_{g} g, \quad B_{\mu}^{(0)}=Z_{B}^{1 / 2} B_{\mu}
$$

then

$$
Z_{g} Z_{B}^{1 / 2}=1
$$

This property is very important for the BPT and the Field Correlator Method in general. Indeed the combinations entering in the latter have the form

$$
\Delta_{n} \equiv \operatorname{tr}\left\langle g F_{\mu_{1} \nu_{1}}^{(B)}(1) \Phi(1,2) g F_{\mu_{2} \nu_{2}}^{(B)}(2) \Phi(2,3) \ldots g F_{\mu_{n} \nu_{n}}^{(B)}(n) \Phi(n, 1)\right\rangle_{B}
$$

where $F_{\mu_{i} \nu_{i}}^{(B)}$ is made of the background field $B_{\mu}$ only, and parallel transporters $\Phi(i, k)$ making Eq.(23) gauge invariant depend also on $g B_{\mu}$. Hence all expression in (23) contains only combinations $g B_{\mu}$, which according to (22) are renorminvariant.

Therefore in all RG relations, in particular in Ovsyannikov-Callan-Simanzyk (OCS) equations the background fields (averaged as in $\Delta_{n}$ or nonaveraged) enter on the same ground as the external momenta, and hence all relations can be kept intact.

E.g. OCS equations for some physical amplitude $\Gamma$ now look like [14].

$$
\left\{-\frac{\partial}{\partial \log \lambda}+\beta \frac{\partial}{\partial g^{2}}+m\left(\gamma_{m}-1\right) \frac{\partial}{\partial m}-\gamma_{\Gamma}\right\} \Gamma\left(\lambda p_{1}, \ldots \lambda p_{N}, \lambda^{2 k} \Delta_{k}, g, \nu\right)=0
$$

where we have defined as usual

$$
\frac{d \alpha_{s}(\lambda)}{d \log \lambda}=\beta\left(\alpha_{s}\right)
$$


and similarly for $\gamma_{m}$, with boundary conditions

$$
\alpha_{s}(\lambda=1)=\alpha_{s}(\nu) .
$$

In writing the r.h.s. of (26) we tacitly assume that $\alpha_{s}$ depends also on all external parameters $\left\{p_{i}\right\},\left\{\Delta_{k}\right\}$ in addition to the dimensional regularization parameter $\nu$ (or its equivalent in other schemes $\mu$ ). The reason why external parameters are not usually written lies in the fact that RG equations (like (24), 25)) define only dependence on one scale parameter, e.g. $\lambda$ and through it on $\nu(\mu)$, while dependence on external parameters is not fixed, and they may in principle enter $\alpha_{s}$ in arbitrary dimensionless combinations, unless additional information is obtained from perturbation expansion (BPT or SPT).

Several words should be said about the choice of renormalization scheme for BPT.

It is convenient to use the $\overline{M S}$ scheme, since also in the presence of background the modified Feynman amplitudes of BPT are diverging at small distances (large momenta) and, when these momenta are much larger than the average magnitude of the background fields, one can neglect the latter, e.g. using OPE one expects corrections of the form $\frac{g^{2}\left\langle F_{\mu \nu}^{2}\right\rangle}{p^{4}}$ which do not alter $Z$ factors. In particular the difference $\Gamma-\Gamma_{\text {pert }} \equiv \Delta \Gamma$, where $\Gamma_{\text {pert }}$ is a usual sum of Feynman amplitudes without background, is UV convergent, so that all UV divergencies of $\Gamma$ are the same as in $\Gamma_{\text {pert }}$.

Now we turn to the possible solutions of Eq. (25). The SPT expansion for $\beta\left(\alpha_{s}\right)$ looks like

$$
\beta^{\text {pert }}\left(\alpha_{s}\right)=-\frac{b_{0}}{2 \pi} \alpha_{s}^{2}-\frac{b_{1}}{4 \pi^{2}} \alpha_{s}^{3}-\frac{b_{2}}{64 \pi^{3}} \alpha_{s}^{4}-\frac{b_{3} \alpha_{s}^{5}}{(4 \pi)^{4}}-\ldots
$$

where the first coefficients are円

$$
\begin{gathered}
S U(3) ; n_{f}=0 ; b_{0}=11 ; b_{1}=51, b_{2}(\overline{M S})=2857, b_{3}(\overline{M S})=58486 \\
S U\left(N_{c}\right) ; n_{f}=0 ; b_{0}=\frac{11}{3} N_{c} ; b_{1}=\frac{17}{3} N_{c}^{2}
\end{gathered}
$$

Following (27) it is reasonable to look for $\beta\left(\alpha_{s}\right)$ in the form

$$
\beta\left(\alpha_{s}\right)=-\frac{b_{0}}{2 \pi} \frac{\alpha_{s}^{2}}{\left[1-\frac{b_{1}}{2 \pi b_{0}} \varphi^{\prime}\left(\frac{1}{\alpha_{s}}\right)\right]}
$$

\footnotetext{
${ }^{1}$ Note that definition of our coefficients $b_{i}$ differ from that in [1] and coincide with [30]
} 
where $\varphi(x)$ is an unknown function and $\varphi^{\prime}(x)$ is the derivative in the argument $x$. Solving (30) for $\alpha_{s}$ one obtains

$$
\alpha_{s}=\frac{4 \pi}{b_{0}\left[\ln \mu^{2} C^{2}+\frac{2 b_{1}}{b_{0}^{2}} \varphi\left(\frac{1}{\alpha_{s}}\right)\right]}
$$

where we have replaced $\lambda \rightarrow \mu$, and $C^{2}$ is an arbitrary function of external parameters of dimension (mass) ${ }^{-2}$.

¿From (27) one concludes that the only condition on $\varphi$ is the expansion in powers of $\alpha_{s}$

$$
\frac{1}{1-\frac{b_{1}}{2 \pi b_{0}} \varphi^{\prime}\left(\frac{1}{\alpha_{s}}\right)}=1+\frac{b_{1}}{2 \pi b_{0}} \alpha_{s}+\frac{b_{2}}{32 \pi^{2} b_{0}} \alpha_{s}^{2}+\frac{b_{3} \alpha_{s}^{3}}{128 \pi^{3} b_{0}} .
$$

One can rewrite (31) in the form

$$
\chi\left(Q^{2}\right)=z-\frac{b_{1}}{2 \pi b_{0}} \varphi(z)
$$

where $z \equiv 1 / \alpha_{s}$ and $\chi\left(Q^{2}\right) \equiv \frac{b_{0}}{4 \pi} \ln \mu^{2} C^{2}$, and $Q^{2}$ is the representative of the external parameters.

As a simple example one can consider the self-energy photon function $\Pi\left(Q^{2}\right)$ entering in the $e^{+} e^{-}$annihilation process, in which case $Q^{2}$ is the only external momentum, while background fields create masses of the bound state spectrum. In this case one can take the form appearing in the lowest approximation of BPT [14], leading to the replacement

$$
\left\{\begin{array}{l}
\ln \mu^{2} C^{2}=\ln \frac{m^{2}}{\Lambda^{2}}+\psi\left(\frac{Q^{2}+M_{0}^{2}}{m^{2}}\right) \\
\Lambda^{2}=\nu^{2} e^{-\frac{4 \pi}{b_{0}} \alpha_{s}\left(\nu^{2}\right)}
\end{array}\right.
$$

Here $\psi(x) \equiv \frac{\Gamma^{\prime}(x)}{\Gamma(x)}$ is the Euler function, having only simple poles at $x=-n, n=0,1,2, \ldots$

This example clearly shows that $\chi\left(Q^{2}\right)$ is a meromorphic function of $Q^{2}$, and we must require that $\alpha_{s}$ be also a meromorphic function of $Q^{2}$, analytic in the Euclidean region $\operatorname{Re} Q^{2} \geq 0$, since otherwise the BPT would violate the analytic properties of amplitudes in the limit $N_{c} \rightarrow \infty$.

In this way we are lead to the conclusion that $\varphi(z)$ should be a meromorphic function of $z=\frac{1}{\alpha_{s}}$, since in this case the r.h.s. of Eq.(32) is a meromorphic function of $Q^{2}$ (meromorphic function of a meromorphic function of 
some argument is again meromorphic). Thus one can equalize functions on both sides of Eq.(32) since both have the same analytic properties.

We do not specify at this point the character of possible singularities; the resulting physical amplitude should have only simple poles at bound state energies, while any finite series of BPT may have poles of higher order and additional poles, as will be seen in examples below.

To illustrate possible forms of $\varphi(z)$ let us consider one example of meromorphic function, namely

$$
\varphi\left(\frac{1}{\alpha_{s}}\right)=\psi\left(\frac{1}{\alpha_{s}}+\Delta\right), \Delta=\text { const. }
$$

Perturbative expansion of $\psi$ has the form

$$
\psi^{\prime}(z)=\frac{1}{z}+\frac{1}{2 z^{2}}+\frac{1}{6 z^{3}}+\ldots, \quad z \rightarrow \infty
$$

or applying it to (35) one has

$$
\psi^{\prime}\left(\frac{1}{\alpha_{s}}+\Delta\right)=\frac{\alpha_{s}}{1+\alpha_{s} \Delta}+\frac{1}{2} \frac{\alpha_{s}^{2}}{\left(1+\alpha_{s} \Delta\right)^{2}}+\frac{\alpha_{s}^{3}}{6\left(1+\alpha_{s} \Delta\right)^{3}}+\ldots
$$

Therefore one obtains

$$
\begin{gathered}
\frac{1}{1-\frac{b_{1}}{2 \pi b_{0}} \psi^{\prime}\left(\frac{1}{\alpha_{s}}+\Delta\right)}=1+\frac{b_{1}}{2 \pi b_{0}} \alpha_{s}+\frac{b_{1}}{2 \pi b_{0}} \alpha_{s}^{2}\left[-\Delta+\frac{1}{2}+\frac{b_{1}}{2 \pi b_{0}}\right]+ \\
+\frac{b_{1}}{2 \pi b_{0}} \alpha_{s}^{3}\left[\Delta^{2}-\Delta+\frac{1}{6}+\left(\frac{b_{1}}{2 \pi b_{0}}\right)(1-2 \Delta)+\left(\frac{b_{1}}{2 \pi b_{0}}\right)^{2}\right]
\end{gathered}
$$

¿From (38) one can find the "theoretical" value of $b_{2}$ and $b_{3}$, namely

$$
\begin{gathered}
b_{2}^{(t h)}=16 \pi b_{1}\left(\frac{1}{2}-\Delta+\frac{b_{1}}{2 \pi b_{0}}\right)=3173.4-16 \pi b_{1} \Delta \\
b_{3}^{(t h)}=64 \pi^{2} b_{1}\left[\Delta^{2}-\Delta+\frac{1}{6}+2 \gamma\left(\frac{1}{2}-\Delta\right)+\gamma^{2}\right], \quad \gamma=\frac{b_{1}}{2 \pi b_{0}} .
\end{gathered}
$$

Now since $\psi(z)$ has poles at $z=0,-1,-2, \ldots, \psi\left(\frac{1}{\alpha_{s}}+\Delta\right)$ has poles at the values of $\alpha_{s}$

$$
\alpha_{s}=-\frac{1}{\Delta},-\frac{1}{1+\Delta},-\frac{1}{2+\Delta}, \ldots
$$


Correspondingly $\psi^{\prime}(z)$ has poles of second order, and as a consequence of (30) $\beta\left(\alpha_{s}\right)$ has zeros at the values of $\alpha_{s}$ given in (40). These zeros condense to $\alpha_{s}=0$ from the negative side.

Poles of $\beta\left(\alpha_{s}\right)$ are defined by the zeros of the denominator in (30), i.e. given by

$$
1=\frac{b_{1}}{2 \pi b_{0}} \psi^{\prime}\left(\frac{1}{\alpha_{s}}+\Delta\right) .
$$

For $\Delta>0$ and $\alpha_{s}>0$ there is one root of (41) at $\alpha_{s}=\alpha_{s}^{(\Delta)}$, if $\Delta \leq \Delta_{0}$ where $\Delta_{0}$ is defined by

$$
\psi^{\prime}\left(\Delta_{0}\right)=\frac{2 \pi b_{0}}{b_{1}}=1.355 ; \Delta_{0}=1.145 .
$$

For $\Delta<\Delta_{0}$ one obtains one pole of $\beta\left(\alpha_{s}\right)$ given by (41), while for $\Delta>\Delta_{0}$ there are no poles of $\beta\left(\alpha_{s}\right)$ for $\alpha_{s} \geq 0$, and $\beta\left(\alpha_{s}\right)$ is monotonically decreasing function.

For $\alpha_{s}<0$ one has infinite number of poles of $\beta\left(\alpha_{s}\right)$ which lie between zeros of $\beta\left(\alpha_{s}\right)$, described by Eq. (40).

We now come to the analytic properties of $\alpha_{s}\left(Q^{2}\right)$, having in mind parametrization (34) for $\Pi\left(Q^{2}\right)$ in $e^{+} e^{-}$annihilation.

Poles of $\alpha_{s}$ are to be found from the equation

$$
\ln \frac{m^{2}}{\Lambda^{2}}+\psi\left(\frac{Q^{2}+M_{0}^{2}}{m^{2}}\right)+\frac{2 b_{1}}{b_{0}^{2}} \psi\left(\frac{1}{\alpha_{s}}+\Delta\right)=0
$$

and we require that for $\operatorname{Re} Q^{2} \geq 0$ there should be no poles of $\alpha_{s}\left(Q^{2}\right)$, which gives condition

$$
\psi\left(\frac{M_{0}^{2}}{m^{2}}\right)+\ln \frac{m^{2}}{\Lambda^{2}}+\frac{2 b_{1}}{b_{0}^{2}} \min \psi\left(\frac{1}{\alpha_{s}}+\Delta\right) \geq 0
$$

Now $\psi(z)>0$ for $z>1.46$, and for $m^{2}=M_{0}^{2}=1 \mathrm{GeV}^{2} ; \Lambda=0.37 \mathrm{GeV}$ (the accurate calculations in [4, 15, 16] strongly prefer $M_{0}=1 \mathrm{GeV}$ ) one obtains for the sum of two first terms in (43) the value

$$
\ln \frac{m^{2}}{\Lambda^{2}}+\psi\left(\frac{M_{0}^{2}}{m^{2}}\right)=1.99-0.577=1.41
$$

Finally one can see that for $\Delta>\Delta_{0}$ one has $\psi(\Delta)>-0.5$ and the l.h.s. of (44) is positive, and hence for all $Q^{2}>0$ also 1.h.s. of Eq.(43) is always 
positive. In this way we have proved that $\alpha_{s}\left(Q^{2}\right)$ has no poles for all $Q^{2}>0$ if $\Delta>\Delta_{0}$.

The resulting form of $\alpha_{s}\left(Q^{2}\right)$ valid in all $Q^{2}$ plane is now

$$
\alpha_{B}=\frac{4 \pi}{b_{0}\left[\ln \frac{m^{2}}{\Lambda^{2}}+\psi\left(\frac{Q^{2}+M_{0}^{2}}{m^{2}}\right)+\frac{2 b_{1}}{b_{0}^{2}} \psi\left(\frac{1}{\alpha_{B}}+\Delta\right)\right]}
$$

where $\Delta>\Delta_{0}=1.145$. For $Q^{2}<0$ the function $\alpha_{s}\left(Q^{2}\right)$ in (46) has zero's and poles which not necessarily coincide with poles of $\psi\left(\frac{Q^{2}+M_{0}^{2}}{m^{2}}\right)$.

But this is as it should be, since the amplitude $\Pi\left(Q^{2}\right)$ must have poles at the final positions

$$
-Q^{2}=\left(M^{(n)}\right)^{2}, \quad n=0,1,2, \ldots
$$

which take into account valence (and Coulomb) gluon effects.

\section{A simple model for constructing perturba- tion series}

In this section we shall consider a simple model which is to illustrate the analytic properties of separate terms of BPT and of its infinite sum. Our discussion in this section will be formally similar to the lectures [20] where old-fashioned perturbation theory was used for SPT, except that we use confined states instead of free quarks and gluons. To this end we define a quantum-mechanical Hamiltonian $H_{0}$ which produces bound states (equivalent of infinite number of mesonic states) and two second-quantized operators $V_{1}(t)$ and $V_{2}(t)$ which create additional valence gluons, to be bound together with quark and antiquark in a sequence of hybrid states. We define the total Hamiltonian

$$
H=H_{0}+V_{1}(t)+V_{2}(t)
$$

and

$$
\begin{gathered}
V_{1}(t)=e^{i \omega_{1} t} v_{1} a^{+}+h . c . \\
V_{2}(t)=e^{i \omega_{2} t} v_{2} b^{+} a^{+} a+h . c .
\end{gathered}
$$

where $a, a^{+}$and $b, b^{+}$are annihilation (creation operators). We do not specify the $d$-space dependence of $v_{1}(x), v_{2}(x) ; t$ - is the common time of the instant 
form of dynamics. It is clear that $V_{1}, V_{2}$ are prototypes of the terms $\bar{\psi} \hat{a} \psi$ and $a^{2} \partial a$ in the QCD Lagrangian, here to simplify matter we disregard in $a_{\mu}(\mathbf{x})$ or $A_{\mu}(\mathbf{k})$ dependence on polarization $\mu$ and momentum $\mathbf{k}$, but introduced $b, a$ to distinguish gluon emission by quarks (in $V_{1}$ ) and by gluons (in $V_{2}$ ).

We assume that $H_{0}$ has only discrete spectrum of mesons (confinement), so for the Green's function we have

$$
i \frac{\partial}{\partial t} G_{0}(t)=H_{0}(t) G_{0} ; \quad G_{0}(t)=\sum_{n} \varphi_{n}^{(0)} \varphi_{n}^{(0)+} e^{-i E_{n}^{(0)} t}
$$

while for the Fourier transform one has

$$
G_{0}(E)=\int_{0}^{\infty} e^{-i E T} G_{0}(t) d t=i \sum_{n} \frac{\varphi_{n}^{(0)} \varphi_{n}^{(0)+}}{E-E_{n}^{(0)}} .
$$

For the full Green's function one can write

$$
i \frac{\partial}{\partial t} G(t)=\left(H_{0}+V(t)\right) G(t), \quad V(t)=V_{1}(t)+V_{2}(t)
$$

with the solution

$$
G\left(q_{1}, q_{2} ; t\right)=<q_{1}\left|e^{-i \int_{0}^{t}\left(H_{0}+V\left(t^{\prime}\right)\right) d t^{\prime}}\right| q_{2}>.
$$

The perturbative series looks like

$$
\begin{gathered}
G(t)=G_{0}+\int G_{0}\left(t-t_{1}\right)(-i) V\left(t_{1}\right) G_{0}\left(t_{1}\right) d t_{1}+ \\
+\iint G_{0}\left(t-t_{1}\right)(-i) V\left(t_{1}\right) d t_{1} G_{0}\left(t_{1}-t_{2}\right)(-i) V\left(t_{2}\right) G_{0}\left(t_{2}\right) d t_{2}+\ldots
\end{gathered}
$$

For the energy-dependent Green's function $G(E)$ the perturbative series can be written taking into account the vacuum state for $a, b$ operators

$$
a|v a c>=b| v a c>=0
$$

which yields

$$
\begin{gathered}
G(E)=G_{0}(E)+G_{0}(E)\left(-i \tilde{V}_{1}\right) G_{0}\left(E-\omega_{1}\right)\left(-i \tilde{V}_{1}\right) G_{0}(E) \\
+G_{0}(E)\left(-i \tilde{V}_{1}\right) G_{0}\left(E-\omega_{1}\right)\left(-i \tilde{V}_{2}\right) G_{0}\left(E-\omega_{1}-\omega_{2}\right)\left(-i \tilde{V}_{2}\right) G_{0}\left(E-\omega_{1}\right)\left(-i \tilde{V}_{1}\right) G_{0}(E)+ \\
+G_{0}(E)\left(-i \tilde{V}_{1}\right) G_{0}\left(E-\omega_{1}\right)\left(-i \tilde{V}_{1}\right) G_{0}(E)\left(-i \tilde{V}_{1}\right) G_{0}\left(E-\omega_{1}\right)\left(-i \tilde{V}_{1}\right) G_{0}(E)+
\end{gathered}
$$


$+G_{0}(E)\left(-i \tilde{V}_{1}\right) G_{0}\left(E-\omega_{1}\right)\left(-i \tilde{V}_{1}\right) G_{0}\left(E-2 \omega_{1}\right)\left(-i \tilde{V}_{1}\right) G_{0}\left(E-\omega_{1}\right)\left(-i \tilde{V}_{1}\right) G_{0}(E)+\ldots$

If one introduces the "gluon propagator"

$$
K_{1}(E)=\left(-i \tilde{V}_{1}\right) G_{0}\left(E-\omega_{1}\right)\left(-i \tilde{V}_{1}\right)
$$

then the partial summation of diagrams with gluons emitted by quarks only yields

$$
G^{\left(K_{1}\right)}(E)=G_{0}(E)+G_{0}(E) K_{1}(E) G_{0}(E)+\ldots=G_{0}(E) \frac{1}{1-K_{1} G_{0}} .
$$

Similarly for the "gluon-b" propagator

$$
K_{2}(E)=\left(-i \tilde{V}_{2}\right) G_{0}\left(E-\omega_{1}-\omega_{2}\right)\left(-i \tilde{V}_{2}\right)
$$

and the "full gluon propagator"

$$
\begin{gathered}
G^{\left(K_{2}\right)}(E)=K_{1}(E)+\left(-i \tilde{V}_{1}\right) G_{0}\left(E-\omega_{1}\right)\left[1+K_{2} G_{0}\left(E-\omega_{1}\right)+\right. \\
\left.\left(K_{2} G_{0}\left(E-\omega_{1}\right)\right)^{2}+\ldots\right]\left(-i \tilde{V}_{1}\right)=\left(-i \tilde{V}_{1}\right) G_{0}\left(E-\omega_{1}\right) \frac{1}{1-K_{2} G_{0}\left(E-\omega_{1}\right)}\left(-i \tilde{V}_{1}\right)
\end{gathered}
$$

One should note that $\tilde{V}_{i}$ are operators and denominators in (59) and (61) are formal operator expressions. Now to analyze the perturbative expansion (57) or its partial sums (59),(61) one should specify the unperturbed spectrum (52) and define matrix elements of $\tilde{V}_{i}$ between unperturbed eigenfunctions $\varphi_{n}^{(0)}$. We shall assume for simplicity that that the spectrum is linear in $n$,

$$
E_{n}^{(0)}=M_{0}+m n, n=0,1, \ldots
$$

and for $V_{n k}^{(i)} \equiv\left(\varphi_{n}^{+(0)} \tilde{V}_{i} \varphi_{k}^{(0)}\right)$ one can assume that at large $n, k$ this matrix element factorizes, $V_{n k}^{(i)}=c_{n}^{+(i)} c_{k}^{(i)}$.

As a consequence one has

$$
G^{\left(K_{1}\right)}(E)=\sum_{n} \frac{\varphi_{n} c_{n}^{(1)+}}{E-E_{n}} \Omega_{1}\left(E-\omega_{1}\right) \frac{1}{\left(1-g(E) \Omega_{1}\left(E-\omega_{1}\right)\right)} \sum_{n} \frac{c_{n^{\prime}}^{(1)} \varphi_{n^{\prime}}^{+}}{E-E_{n^{\prime}}}
$$

where we have defined

$$
\Omega_{1}\left(E-\omega_{1}\right) \equiv-\sum_{k} \frac{\left|c_{k}^{(1)}\right|^{2}}{E-E_{k}-\omega_{1}}
$$




$$
g(E) \equiv \sum_{n} \frac{\left|c_{n}^{(1)}\right|^{2}}{E-E_{n}}
$$

It is clear that $\Omega_{1}\left(E-\omega_{1}\right)$ has poles at the "gluon excited" i.e. "hybrid state" masses $E=E_{k}+\omega_{1}$, however the partial sum $G^{\left(K_{1}\right)}(E)$ has shifted poles due to vanishing of denominator in (63), in addition to unperturbed poles at $E_{n}$.

A similar situation occurs for $G^{\left(K_{2}\right)}(E)$. If one defines

$$
\begin{gathered}
\Omega_{2}\left(E-\omega_{1}\right)=\sum_{k} \frac{\left|c_{k}^{(2)}\right|^{2}}{E-E_{k}-\omega_{1}} \\
\Pi_{2}\left(E-\omega_{1}-\omega_{2}\right)=-\sum \frac{\left|c_{k}^{(2)}\right|^{2}}{E-E_{k}-\omega_{1}-\omega_{2}}
\end{gathered}
$$

then one can rewrite (61) as

$$
G^{\left(K_{2}\right)}(E)=\frac{\left(-i \tilde{V}_{1}\right) \mid \varphi_{n}>}{E-E_{n}-\omega_{1}}\left\{\delta_{m n}+c_{n}^{+(2)} \frac{\Pi_{2}\left(E-\omega_{1}-\omega_{2}\right) c_{m}^{(2)}}{1-\Omega_{2}\left(E-\omega_{1}\right) \Pi_{2}\left(E-\omega_{1}-\omega_{2}\right)}\right\} \frac{<\varphi_{m} \mid\left(-i \tilde{V}_{1}\right)}{E-E_{m}-\omega_{1}} .
$$

This exercise was undertaken to illustrate that the "full gluon propagator" $G^{\left(K_{2}\right)}(E)$ has in addition to unperturbed "one-gluon poles" $\frac{1}{E-E_{n}-\omega_{1}}$ also shifted "two-gluon poles" due to vanishing of denominator in (68), but not two-gluon poles" themselves.

To make our model more realistic and finally to incorporate in it QCD features, one should make several steps. Firstly, one must take into account negative energy states, corresponding to the background in time motion, i.e. as in the Feynman propagator we replace (cf. 20])

$$
\frac{1}{E-E_{n}} \rightarrow \frac{1}{E^{2}-E_{n}^{2}} \rightarrow \frac{1}{s-\left(M_{n}^{(0)}\right)^{2}}
$$

Correspondingly, the hybrid propagators take the form

$$
\frac{1}{E-\omega_{1}-E_{n}} \rightarrow \frac{1}{s-\left(M_{n}^{(1)}\right)^{2}}, \quad \frac{1}{E-\omega_{1}-\omega_{2}-E_{n}} \rightarrow \frac{1}{s-\left(M_{n}^{(2)}\right)^{2}}
$$

where $M_{n}^{(1)}, M_{n}^{(2)}$ are hybrid masses with one and two gluons respectively.

Now one must take into account the structure of $\tilde{V}_{1}, \tilde{V}_{2}$ operators in QCD. Both describe emission of one gluon from quark and gluon line respectively, 
and both are local field operators, whereas wave functions refer to the nonlocal instantaneous bound state objects, namely, mesons $(q \bar{q})$, 1-gluon hybrid $(q \bar{q} g)$ and 2-gluon hybrid $(q \bar{q} g g)$. We shall denote the full set of the corresponding eigenfunctions as $\varphi_{n}^{(0)}(\mathbf{x}), \varphi_{n, \nu_{1}}^{(1)}(\mathbf{x}, \mathbf{y})$ and $\varphi_{n, \nu_{1} \nu_{2}}^{(2)}(\mathbf{x}, \mathbf{y}, \mathbf{z})$. Note that the string pieces between quarks and gluons formed by background field are straight lines, given by vectors $\mathbf{x}, \mathbf{y}$ in $\varphi_{n}^{(1)}$, and by $\mathbf{x}, \mathbf{y}, \mathbf{z}$ in $\varphi_{n}^{(2)}$, and they contribute to the rotational and vibrational eigenenergies. We also note that the string vibration in this background picture is described by the full set of one-, two- and many-gluon hybrid states.

Here we have introduced in hybrid eigenfunctions additional lower indices $\nu_{1}$ and $\nu_{1}, \nu_{2}$ to describe the quantum numbers of additional bound gluons, note that $\nu_{i}$ are multidimensional vectors with integer components. Correspondingly hybrid masses depend on those quantum numbers;

$$
\begin{gathered}
M_{n}^{(0)}=M^{(0)}\left(n_{r}, L ; J^{P C}\right) \\
M_{n}^{(1)}=M^{(1)}\left(n_{r}, L ; n_{r}^{\prime}, L^{\prime} ; J^{P C}\right) \\
M_{n}^{(2)}=M^{(2)}\left(n_{r}, L, n_{r}^{\prime}, L^{\prime}, n_{r}^{\prime \prime}, L^{\prime \prime} ; J^{P C}\right) .
\end{gathered}
$$

Here $n_{r} L$ refer to the radial quantum number and angular momentum of quark with respect to antiquark, each additional gluon has its own $n_{r}, L$ with respect to neighboring quark or gluon. We have not taken into account yet spin degrees of freedom of $q, \bar{q}$ and gluons, which create fine and hyperfine structure of levels, and we shall neglect those, since we are interested in the dominant contributions to the masses at large excitations, and our final goal is the result of summation over all excitations in $G(E)$ and establishing of some relation between $\mathrm{SPT}$ and $\mathrm{BPT}$, which requires analysis of high excited states in the sums.

Now the local character of the operators $\tilde{V}_{1}, \hat{V}_{2}$ mentioned above leads to the following properties of matrix elements (since $\tilde{V}_{i}$ acts only at one of the end points of vectors $\mathbf{x y}$, it does not influence quantum wave function, unless $\tilde{V}_{i}$ contains derivative in coordinate as in $\tilde{V}_{2}$, and in this case it changes quantum numbers by a discrete number):

$$
\begin{gathered}
<n_{r}, L\left|\tilde{V}^{(1)}\right| \bar{n}_{r}, \bar{L} ; n_{r}^{\prime}, L^{\prime}>\sim \delta_{n_{r} \bar{n}_{r}} \delta_{L \bar{L}} \\
<n_{r}, L ; n_{r}^{\prime}, L^{\prime}\left|\tilde{V}^{(2)}\right| \bar{n}_{r}, \bar{L}, \bar{n}_{r}^{\prime}, \bar{L}^{\prime} ; n_{r}^{\prime \prime}, L^{\prime \prime}>\sim \delta_{n_{r} \bar{n}_{r}} \delta_{L \bar{L}} \delta_{\bar{n}_{r}^{\prime} n_{r}^{\prime}} \delta_{\bar{L}^{\prime} L^{\prime}} .
\end{gathered}
$$

Moreover we shall assume that $\left(M_{n}^{(i)}\right)^{2}$ depends on all quantum numbers linearly,

$$
\left(M_{n}^{(i)}\right)^{2}=m^{2}\left(a \sum_{i} n_{r}^{(i)}+b \sum L^{(i)}\right)
$$


Let us now apply these rules to the computation of the "full gluon propagator" $G_{2}^{K}(E)$ and try to find a correspondence with the standard perturbative calculations.

To this end we assume that the sum entering in $K_{1}(E)$ is converging, i.e.

$$
<n\left|K_{1}(E)\right| m>=\sum_{n, \nu} \frac{<n\left|-i \tilde{V}_{1}\right| \bar{n}, \nu><\bar{n}, \nu\left|-i \tilde{V}_{1}\right| m>}{Q^{2}+M^{2}(\bar{n}, \nu)} \sim \frac{1}{Q^{2}}
$$

where we denote $n(\nu)=n_{r}, L\left(n_{r}^{\prime}, L^{\prime}\right)$ and replace $s \rightarrow-Q^{2}$.

The same type of expression for $K_{2}(E)$ yields

$$
<n, \nu\left|K_{2}(E)\right| \tilde{n}, \tilde{\nu}>=\sum_{\bar{n}, \bar{\nu}, \nu^{\prime}} \frac{<n, \nu\left|-i \tilde{V}_{2}\right| \bar{n}, \bar{\nu}, \nu^{\prime}><\bar{n}, \bar{\nu}, \nu^{\prime}\left|-i \tilde{V}_{2}\right| \tilde{n}, \tilde{\nu}>}{Q^{2}+M^{2}\left(\bar{n}, \bar{\nu}, \nu^{\prime}\right)} .
$$

Due to the properties (75) the sum in (77) is actually a double sum in, say, $L_{\nu}^{\prime}, n_{r}^{\prime}$, while $M^{2}$ depends on them linearly and we assume at this point that coefficients in (77) do not depend on $\nu^{\prime}$. Therefore one must regularize this expression, making one subtraction at $s=0$, and renormalize the retaining logarithmic divergence. This is in full correspondence with the standard perturbative calculation of one gluon loop correction to gluon propagator. As a result $K_{2}(E)$ aquires the form

$$
<n, \nu\left|k_{2}(E)\right| \tilde{n}, \tilde{\nu}>\sim Q^{2} \ln \left(\frac{Q^{2}-M^{2}(n, \nu)-\delta M^{2}}{\mu^{2}}\right) \delta_{n \tilde{n}} \delta_{\nu \tilde{\nu}}
$$

where

$$
\delta M^{2}=M_{m i n}^{2}\left(n, \nu, \nu^{\prime}\right)-M^{2}(n, \nu)
$$

and minimization is taken with respect to $\nu^{\prime}$.

Now one can consider $G_{2}^{K}(E)$ extracting coupling constant $g_{0}$ from $\tilde{V}_{1}, \tilde{V}_{2}$ one has a series (at large $s$ )

$$
G^{\left(K_{2}\right)}(s) \sim \frac{g_{0}^{2}}{Q^{2}}-C \frac{g_{0}^{4}}{Q^{2}} \ln \frac{Q^{2}+\bar{M}^{2}}{\mu^{2}}+\ldots \sim \frac{1}{Q^{2}} \frac{g_{0}^{2}}{\left(1+g_{0}^{2} C \ln \frac{Q^{2}+\bar{M}^{2}}{\mu^{2}}\right)} .
$$

Expression (80) is similar to that of the running coupling constant, but with the mass $\bar{M}^{2}$, corresponding to the freezing coupling constant derived in [12] and studied later in [13, 14]. We note that logarithms in (80) appear as a result of summation over the string like spectrum (75) with the coefficients asymptotically constant, which is the property of matrix elements 
of eigenfunctions of linear interaction [14]. Therefore this property and the correspondence found above can be only an asymptotics, while at finite $s$ there are corrections, which should be taken into account. In the following chapter we look into this problem more carefully.

\section{$5 \quad$ Nonasymptotic terms in the spectral sums}

We start with the definition of the photon vacuum polarization function $\Pi\left(q^{2}\right)$ and after a short review of asymptotic correspondence between the spectral sum and perturbative expansion, we shall look more carefully into the correction terms and comparison between OPE and spectral sum.

We define the photon vacuum polarization function $\Pi\left(q^{2}\right)$ as a correlator of electromagnetic currents for the process $e^{+} e^{-} \rightarrow$ hadrons in usual way

$$
-i \int d^{4} x e^{i q x}<0 \mid T\left(j_{\mu}(x) j_{\nu}(0) \mid 0>=\left(q_{\mu} q_{\nu}-g_{\mu \nu} q^{2}\right) \Pi\left(q^{2}\right),\right.
$$

where the imaginary part of $\Pi$ is related to the total hadronic ratio $R$ as

$$
R\left(q^{2}\right)=\frac{\sigma\left(e^{+} e^{-} \rightarrow \text { hadrons }\right)}{\sigma\left(e^{+} e^{-} \rightarrow \mu^{+} \mu^{-}\right)}=12 \pi e \operatorname{Im} \Pi\left(\frac{q^{2}}{\mu^{2}}, \alpha_{s}(\mu)\right)
$$

There are two standard approaches to calculate $\Pi\left(q^{2}\right)$; first, when purely perturbative expansion is used, which is now known to the order $0\left(\alpha_{s}^{3}\right)[21]$. The second one is the OPE approach [6], which includes the NP contributions in the form of local condensates. For two light quarks of equal masses $\left(m_{u}=\right.$ $\left.m_{d}=m\right)$ it yields for $\Pi\left(Q^{2}\right)$

$$
\frac{1}{e_{q}^{2}} \Pi\left(Q^{2}\right)=-\frac{1}{4 \pi^{2}}\left(1+\frac{\alpha_{s}}{\pi}\right) \ln \frac{Q^{2}}{\mu^{2}}+\frac{6 m^{2}}{Q^{2}}+\frac{2 m<q \bar{q}>}{Q^{4}}+\frac{\alpha_{s}<F F>}{12 \pi Q^{4}}+\ldots
$$

To make explicit the renormalization of $\alpha_{B}$, one can write the perturbative expansion of the function $\Pi\left(Q^{2}\right)(23)$ as

$$
\Pi\left(Q^{2}\right)=\Pi^{(0)}\left(Q^{2}\right)+\alpha_{B} \Pi^{(1)}\left(Q^{2}\right)+\alpha_{B}^{2} \Pi^{(2)}\left(Q^{2}\right)+\ldots
$$

We now again use the large $N_{c}$ approximation, in which case $\Pi^{(0)}$ contains only simple poles in $Q^{2}[27,28]$ :

$$
\Pi^{(0)}\left(Q^{2}\right)=\frac{1}{12 \pi^{2}} \sum_{n=0}^{\infty} \frac{C_{n}}{Q^{2}+M_{n}^{2}}
$$


and the mass $M_{n}$ is an eigenvalue of the Hamiltonian $H^{(0)}$, which contains only quarks and background field $B_{\mu}$,

$$
H^{(0)} \Psi_{n}=M_{n} \Psi_{n}
$$

In what follows we are mostly interested in the long-distance effective Hamiltonian which obtains from $G_{q \bar{q}}$ for large distances, $r \gg T_{g}$, where $T_{g}$ is the gluonic correlation length of the vacuum, $T_{g} \approx 0.2 \mathrm{fm},[19]$ :

$$
G_{q \bar{q}}(x, 0)=\int D B \eta(B) \operatorname{tr}\left(\gamma_{\mu} G_{q}(x, 0) \gamma_{\mu} G_{q}(0, x)\right)=<x\left|e^{-H^{(0)}|x|}\right| 0>
$$

At these distances one can neglect in $G_{q}$ the quark spin insertions $\sigma_{\mu \nu} F_{\mu \nu}$ and use the area law:

$$
<W_{C}>\rightarrow \exp \left(-\sigma S_{\text {min }}\right)
$$

where $S_{\text {min }}$ is the minimal area inside the loop $C$.

Then the Hamiltonian in (87) is readily obtained by the method of [22]. In the c.m. system for the orbital momentum $l=0$ it has the familiar form:

$$
H^{(0)}=2 \sqrt{\vec{p}^{2}+m^{2}}+\sigma r+\text { const }
$$

where constant appears due to the self-energy parts of quarks [23], while for $l=2$ a small correction from the rotating string appears [22], which we neglect in the first approximation.

Now we can use the results of the quasiclassical analysis of $H^{(0)}$ [24] where the values of $M_{n}, C_{n}$ have been already found.

These results can be represented as follows $\left(n=n_{r}+l / 2, n_{r}=0,1,2, \ldots, l=\right.$ $0,2)$,

$$
M_{n}^{2}=2 \pi \sigma\left(2 n_{r}+l\right)+M_{0}^{2},
$$

where $M_{0}^{2}$ is a weak function of quantum numbers $n_{r}, l$ separately, comprising the constant term of $(\sqrt[89]{)}$; in what follows we shall put it equal to the $\rho$-meson mass, $M_{0}^{2} \simeq m_{\rho}^{2}$ (see [23] for more discussion).

For $C_{n}$ one obtains quasiclassically [24]

$$
\begin{gathered}
C_{n}(l=0)=\frac{2}{3} e_{q}^{2} N_{c} m^{2}, \quad m^{2} \equiv 4 \pi \sigma \\
C_{n}(l=2)=\frac{1}{3} e_{q}^{2} N_{c} m^{2}
\end{gathered}
$$


Using asymptotic expressions (90),(91) for $M_{n}, C_{n}$ and starting with $n=n_{0}$, one can write

$$
\begin{gathered}
\Pi^{(0)}\left(Q^{2}\right)=+\frac{1}{12 \pi^{2}} \sum_{n=0}^{n_{0}-1} \frac{C_{n}}{M_{n}^{2}+Q^{2}}-\frac{e_{q}^{2} N_{c}}{12 \pi^{2}} \psi\left(\frac{Q^{2}+M_{0}^{2}+n_{0} m^{2}}{m^{2}}\right) \\
+ \text { divergent constant, }
\end{gathered}
$$

where we have used the equality

$$
\begin{gathered}
\sum_{n=n_{0}}^{\infty} \frac{1}{M_{n}^{2}+Q^{2}}=-\frac{1}{m^{2}} \psi\left(\frac{Q^{2}+M_{0}^{2}+n_{0} m^{2}}{m^{2}}\right) \\
+ \text { divergent constant }
\end{gathered}
$$

and $\psi(z)=\frac{\Gamma^{\prime}(z)}{\Gamma(z)}$.

In (92) we have separated the first $n_{0}$ terms to treat them nonquasiclassically, while keeping for other states with $n \geq n_{0}$ the quasiclassical expressions (90),(91). In what follows, however, we shall put $n_{0}=1$ for simplicity. It was shown in [14] that even in this case our results reproduce $e^{+} e^{-}$experimental data with good accuracy.

Consider now the asymptotics of $\Pi^{(0)}\left(Q^{2}\right)$ at large $Q^{2}$. Using the asymptotics of $\psi(z)$ :

$$
\psi(z)_{z \rightarrow \infty}=\ln z-\frac{1}{2 z}-\sum_{k=1}^{\infty} \frac{B_{2 k}}{2 k z^{2 k}}
$$

where $B_{n}$ are Bernoulli numbers, from (92) one obtains

$$
\Pi^{(0)}\left(Q^{2}\right)=-\frac{e_{q}^{2} N_{c}}{12 \pi^{2}} \ln \frac{Q^{2}+m^{2}}{\mu^{2}}+0\left(\frac{m^{2}}{Q^{2}}\right)
$$

One can easily see that this term coincides at $Q^{2} \gg M_{0}^{2}$ with the first term in OPE (22) - the logarithmic one. Taking the imaginary part of (39) at $Q^{2} \rightarrow-s$ one finds

$$
R\left(q^{2}\right)=12 \pi \operatorname{Im} \Pi^{(0)}(-s)=N_{c} e_{q}^{2},
$$

i.e. it means that we have got from $\Pi^{(0)}$ the same result as for the free quarks. This fact is the explicit manifestation of the quark-hadron duality.

We can now identify $\Pi^{(0)}\left(Q^{2}\right)$ as

$$
\Pi^{(0)}\left(Q^{2}\right)=-\frac{e_{q}^{2} N_{c}}{12 \pi^{2}} \psi\left(\frac{Q^{2}+M_{0}^{2}}{m^{2}}\right)
$$


and compare this expression with the OPE series (83).

To this end one can use the asymptotic expansion of $\psi(x)$

$$
\begin{gathered}
\psi(z)_{z \rightarrow \infty}=\ln z-\frac{1}{2 z}-\sum_{k=1}^{\infty} \frac{B_{2 k}}{2 k z^{2 k}}= \\
=\ln z-\frac{1}{2 z}-\frac{1}{12 z^{2}}+\frac{1}{120 z^{4}}-\frac{1}{252 z^{6}}+\ldots
\end{gathered}
$$

and the expansion of

$$
\psi(z)=-C-\sum_{k=0}^{\infty}\left(\frac{1}{z+k}-\frac{1}{k+1}\right), \quad C=0.577 .
$$

Two features are immediately seen when comparing expansion (98) with the OPE (83) (see also discussion in 13, 14]) i) for $m=0$ the term $O\left(1 / Q^{2}\right)$ is absent, since one cannot construct a local gauge-invariant operator of dimension mass squared. One should stress, that this type of operator may appear from the interference of perturbative and nonperturbative contributions [25] and is welcome on phenomenological grounds [26]. However in the framework of BPT accepted in this paper the term $\Pi^{(0)}\left(Q^{2}\right)$ is solely due to NP contributions, and cannot have OPE terms $O\left(1 / Q^{2}\right)$.

ii) The generic terms in $\Pi^{(0)}\left(Q^{2}\right)$ from expansion (97) have the magnitude $\frac{m^{2 n}}{Q^{2 n}}$, while the corresponding OPE terms are much smaller, e.g. one should compare $-\frac{\pi}{3} \frac{\alpha_{s}\langle F F\rangle}{Q^{4}} \approx-\frac{0.04 \mathrm{GeV}^{4}}{Q^{4}}(\mathrm{OPE})$ with $-\frac{m^{4}}{12 Q^{4}} \approx-\frac{0.5 \mathrm{GeV}^{4}}{Q^{4}}$ (spectral decomposition (97)).

In the remaining part of this section we shall discuss possible solutions of problems i) and ii).

Concerning the point i), one should say that (97) is an approximate expression for $\Pi^{(0)}$ valid for large $Q^{2}$, i.e. reproducing correctly the logarithmic term in (95). However, if one interested in the next asymptotic terms, one should take into account corrections to $C_{n}$ and $M_{n}$ in (92).

The first major correction comes from the fact that we have taken into account in (93) that levels with $n_{r}+1, l=0$ and $n_{r}, l=2$ are degenerate (in the lowest approximation when one neglects spin-dependent force) and hence used the sum of coefficients (91) for $C_{n}$ in (92). However it is not true for the $\rho$-meson, where coefficient with $l=0$ yields $\frac{2}{3}$ of $C_{n}$. Therefore one should use instead of $\psi(z)$ in (93), (97) the corrected expression

$$
\psi(z) \rightarrow \tilde{\psi}(z) \equiv \psi(z)+\frac{1-\gamma}{z}
$$


where $\frac{1-\gamma}{z}$ cancels the corresponding pole (with $k=0$ ) in $(99)$ and replaces it with the correct coefficient $\gamma=\gamma_{0}=\frac{2}{3}$.

Moreover, in the analysis [14] it was realized that the value $\gamma_{0}=\frac{2}{3}$ (which does not take into account radiative correction to the $\rho$ width) yields some $10 \%$ discrepancy in the leptonic width of $\rho$ meson. Now the destiny of the $\frac{1}{q^{2}}$ term in the asymptotic expansion (98) depends on the exact value of $\gamma$. Indeed, using (98), one obtains the expansion

$$
\tilde{\psi}(z)=\ln z+\frac{1}{z}\left(1-\gamma-\frac{1}{2}\right)+O\left(\frac{1}{z^{2}}\right)
$$

where $z=\frac{Q^{2}+M_{0}^{2}}{m^{2}} \rightarrow \frac{Q^{2}}{m^{2}}$.

One can see that the requirement of the absence of $O\left(1 / Q^{2}\right)$ terms implies the following condition of $\gamma$ :

$$
\gamma=\frac{2}{3} \kappa_{\text {rad }}=\frac{1}{2}
$$

where $\kappa_{\text {rad }}=1-\Delta \kappa$, and $\Delta \kappa$ is the radiative correction due to gluon exchanges in the leptonic width of $\rho$-meson. It is clear that condition is in the correct ballpark and one should take into account other possible corrections, to be discussed below.

Till now we have considered the lowest approximation for for $M_{n}^{2}$ and $C_{n}$, Eqs.(90), (91), where $M_{n}^{2}$ is linear in $n$ and $C-n$ is $n$-independent. However, there are corrections to this quasiclassical behaviour [27], yielding

$$
\begin{gathered}
M_{n}^{2}=4 \pi \sigma n+M_{0}^{2}+\frac{b}{n} \\
C_{n}=C_{n}^{(0)}\left(1+\frac{a}{n}\right)
\end{gathered}
$$

where $C_{n}^{(0)}$ is given in (91). To see the effect of these corrections we first put $b=0$ and consider the sum (cf. Eq.(93)), which can be expressed through $\psi(z)$ again

$$
\begin{gathered}
\sum_{n=n_{0}=1}^{\infty} \frac{\left(1+\frac{a}{n}\right)}{M_{0}^{2}+m^{2}+Q^{2}}= \\
-\left\{\frac{1}{m^{2}}\left(1-\frac{a m^{2}}{M_{0}^{2}+Q^{2}}\right) \psi \frac{\left(M_{0}^{2}+Q^{2}+m^{2}\right)}{m^{2}}-C \frac{a}{M_{0}^{2}+Q^{2}}\right\}, C=0.577 .
\end{gathered}
$$


Thus we see that the correction $\frac{a}{n}$ yields a power correction $\frac{a m^{2}}{M_{0}^{2}+Q^{2}}$ which modifies asymptotic expansion of $\psi(z)$ (98) and can occasionally diminish some term to bring them closer in agreement with in the OPE (83). The same is true also for the mass correction $b / n$ in (103), which yields power correction $\frac{b m^{2}}{\left(M_{0}^{2}+Q^{2}\right)^{2}}$ as a factor in expression like (104). However since coefficients in (98) are fast diminishing, the overall cancellation in all terms seems to be unprobable and therefore the problem ii) remains unsolved.

\section{Analytic properties of $\alpha_{B}(s)$}

In this section we shall discuss the analytic structure of the BPT in the limit $N_{c} \rightarrow \infty$ in the whole complex plane of $-s=Q^{2}$ and establish connection between our results and SPT. In what follows the basic role will be played by the function $\Pi\left(Q^{2}\right)$ and we shall use this example as a typical one, generalizing to other amplitudes at the end of this section.

Our starting point is the BPT for $\Pi\left(Q^{2}\right)$, Eq.(84). Analytic properties of $\Pi^{(0)}\left(Q^{2}\right)$ are given in Eq.(85), showing a sequence of purely nonperturbative poles

$$
s=-Q^{2}=\left(M_{n}^{(0)}\right)^{2}, n=0,1,2, \ldots
$$

Inclusion of $\alpha_{B}$ to all orders makes the following changes with the unperturbed spectrum (105). The gluon exchanges play two different roles: a) instantaneous Coulomb interaction shifts NP poles b) gluons $a_{\mu}$ propagating in the confining film form hybrid states. Correspondingly the NP poles are mixed and shifted by the hybrid states. Therefore one has the following hierarchy of the shifts of original NP meson masses

$$
M_{n}^{(0)} \rightarrow M_{n}^{(0)}\left(O\left(\left(\alpha_{B}\right)^{K}\right)\right) \equiv M_{n}^{(0)}(k)
$$

The one-gluon hybrid state (apart from Coulomb shift, which we disregard for simplicity at this moment) with mass $M_{n}^{(1)}$ is also shifted due to mixing with meson and higher hybrid states, the mixing being characterized by the power of $\alpha_{B}$, and therefore one has

$$
M_{n}^{(1)}\left(O\left(\left(\alpha_{B}\right)^{k}\right)\right) \equiv M_{n}^{(1)}(k)
$$

In a similar way one has shifted states $M_{n}^{(m)}(k)$ for m-hybrid configurations (with $m$ gluons). 
As it is clear from the schematic example of section 4, the terms $O\left(g_{0}^{2}\right)$ (the second term on the r.h.s.of (57)) refer to 1-gluon hybrid states and do not contain any analytic structure of $\alpha_{B}\left(Q^{2}\right)$, since they are not yet renormalized (and of course not yet RG improved, i.e.resummed).

This renormalization process, leading to the nontrivial dependence of $\alpha_{B}\left(Q^{2}\right)$, starts with the diagrams of the order $O\left(g_{0}^{4}\right)$ in SPT (and of the order $O\left(\tilde{V}_{1}^{2} \tilde{V}_{2}^{2}\right)$ in the example of section 4 - see the third term on the r.h.s. of $(57))$.

This term and its QCD analog has singularities of the double hybrid (the term $G_{0}\left(E-\omega_{1}-\omega_{2}\right)$ in $\left.(57)\right)$, corresponding to the gluon-loop diagram and accompanying diagrams in $O\left(g_{0}^{4}\right)$ in SPT.

Next one can do a RG improvement of this $O\left(g_{0}^{4}\right)$ result, which leads to the resummation presented in $G_{2}^{K}(E)$, Eq. (68). In this approximation one can write

$$
\alpha_{B}=\frac{4 \pi}{b_{0}\left[\ln \frac{m^{2}}{\Lambda^{2}}+\tilde{\psi}\left(Q^{2}\right)\right]}
$$

where $\tilde{\psi}\left(Q^{2}\right)$ is a meromorphic function with poles at the double-hybrid position, i.e. at

$$
s=M_{n}^{(2)}(k=0)
$$

In the particular case when $\tilde{\psi}\left(Q^{2}\right)=\psi\left(\frac{Q^{2}+M_{0}^{2}}{m^{2}}\right)$ and $M_{0} \equiv M_{0}^{(2)}(k=0)$ the form (108) coincides with the one, suggested in [14].

In the large $Q^{2}$ limit one has

$$
\tilde{\psi}\left(Q^{2}\right) \rightarrow \ln \frac{Q^{2}+M_{0}^{2}}{m^{2}}
$$

and $\alpha_{B}$ has the same freezing form, which was extensively used before in [12]-16]:

$$
\bar{\alpha}_{B}=\frac{4 \pi}{b_{0} \ln \frac{Q^{2}+M_{0}^{2}}{\Lambda^{2}}}
$$

Now we can compare the form (108) with our model solution (46). Indeed when $\frac{Q^{2}+M_{0}^{2}}{m^{2}}$ is large, one can keep in the denominator of (46) the first two terms, and one has the form coinciding with (108). In the next approximation one can keep in (108) the term $\frac{2 b_{1}}{b_{0}^{2}} \psi\left(\frac{1}{\alpha_{B}}+\Delta\right)$ which yields next order approximation, when $M_{n}^{(2)}(0)$ is shifted to the higher order position $M_{n}^{(2)}(2)$ and so on. 
Thus one can see that while the $O\left(g_{0}^{4}\right)$ term contains the double hybrid poles, the RG improvement leads to the shifted poles, which do not correspond to the final physical poles, the latter are the result of infinite resurmmation to all orders.

Let us now discuss the imaginary part of $\Pi\left(Q^{2}\right)$. It is clear that $\Pi^{(0)}\left(Q^{2}\right)$ contains the NP poles. The term $O\left(g_{0}^{2}\right)$ (equivalent of the second term on the r.h.s. of (57)) contains double NP poles from $\left(G_{0}(E)\right)^{2}$ and 1-gluon hybrid poles from $G_{0}\left(E-\omega_{1}\right)$. The $O\left(g_{0}^{4}\right)$ term contains double NP poles, double 1-gluon hybrid poles and 2-gluon hybrid poles - the latter are associated with $\alpha_{B}$ in the lowest order. The same type of classification goes on for higher terms. The outcome for the expansion (84) is that in the term $\alpha_{B}^{k} \Pi^{(k)}\left(Q^{2}\right)$ part of singularities is associated with $\alpha_{B}^{k}$ and another part with $\Pi^{(k)}\left(Q^{2}\right)$.

Thus we see that the requirements of i) logarithmic behaviour of $\tilde{\psi}\left(Q^{2}\right)$ at large $Q^{2}$ and ii) meromorphic analytic properties in the whole $Q^{2}$ plane, leads to the one possible representation of $\tilde{\psi}\left(Q^{2}\right)$;

$$
\tilde{\psi}\left(Q^{2}\right)=\psi\left(\frac{Q^{2}+M_{0}^{2}}{m^{2}}\right)+\sum_{k=0}^{N} \frac{a_{k}}{Q^{2}+m_{k}^{2}}
$$

where $N$ is finite, so that logarithmic asymptotics of $\psi(z)$ is not modified.

Let us now compare our Eq.(31) with the SPT, more explicitly with GellMann-Low equation, written in the form adopted by Radyushkin [9].

$$
L=\ln \frac{Q^{2}}{\Lambda^{2}}=\frac{4 \pi}{b_{0} \alpha_{s}}+\frac{2 b_{1}}{b_{0}^{2}} \ln \frac{\alpha_{s}}{4 \pi}+\tilde{\Delta}+\frac{b_{2} b_{0}-8 b_{1}^{2}}{2 b_{0}^{3}} \frac{\alpha_{s}}{4 \pi}+O\left(\left(\frac{\alpha_{s}}{4 \pi}\right)^{2}\right) .
$$

Here $\tilde{\Delta}$ is a parameter of integration which fixed the definition of $\Lambda$. This should be compared with our Eq.(31) which we can write in a similar form:

$$
\ln \frac{m^{2}}{\Lambda^{2}}+\psi\left(\frac{Q^{2}+M_{0}^{2}}{m^{2}}\right)=\frac{4 \pi}{b_{0} \alpha_{s}}-\frac{2 b_{1}}{b_{0}^{2}} \varphi\left(\frac{1}{\alpha_{s}}\right)
$$

It is clear that our analysis with the expansion (32) is equivalent to the analysis of (113) for large $Q^{2}$ and small $\alpha_{s}$. As we have found in (39) the predicted values of $b_{2}^{(t h)}, b_{3}^{(t h)}$ depend on the value of $\Delta$ and keeping the choice $\varphi\left(\frac{1}{\alpha_{s}}\right)=\psi\left(\frac{1}{\alpha_{s}}+\Delta\right)$, one has from (39) for $\Delta \geq \Delta_{0}=1.145$

$$
b_{2}^{(t h)}<238.15: \quad b_{3}^{(t h)} \geq 18266
$$


To repair obtained values and get agreement with $\overline{M S}$ calculated values 21] one can use the modified $\psi(z)$ function like that in (112), namely

$$
\varphi\left(\frac{1}{\alpha_{s}}\right)=\psi\left(\frac{1}{\alpha_{s}}+\Delta\right)+\sum_{k=0}^{N} \tilde{A}_{k} \frac{\alpha_{s}}{1+\alpha_{s}(\Delta+k)} .
$$

It is important that the position of poles of $\psi(k)$ are not changed, the only change is in the coefficients of those pole terms, which can change the expansion (37), yielding agreement with $\overline{M S}$ coefficients of expansion (27).

We consider now the problem of analytic continuation of $\alpha_{s}\left(Q^{2}\right)$ and $\alpha_{B}\left(Q^{2}\right)$ from the Euclidean region $Q^{2} \geq 0$ to the time-like region $Q^{2}<0$.

One can see that the freezing form $\bar{\alpha}_{B}$ (111) is well defined for $Q^{2} \geq-M_{0}^{2}$, and has a logarithmic branch point at $Q^{2}=-M_{0}^{2}$.

However this is an artefact of representation (112), which is valid in the asymptotics when $\frac{Q^{2}+M_{0}^{2}}{\Lambda^{2}} \gg 1$, otherwise one should use the original form (108).

Choosing it in the form

$$
\alpha_{B}=\frac{4 \pi}{b_{0}\left[\ln \frac{m^{2}}{\Lambda^{2}}+\psi\left(\frac{Q^{2}+M_{0}^{2}}{m^{2}}\right)\right]}
$$

one can see that $\alpha_{B}\left(Q^{2}\right)$ is defined in all complex plane $Q^{2}$ and has only isolated poles there at zeros of the denominator in (117). These poles are the result of partial resummation of two-gluon hybrid poles present in $\psi\left(\frac{Q^{2}+M_{0}^{2}}{m^{2}}\right)$ and lying at $s \equiv-Q^{2}=M_{0}^{2}+n m^{2}, n=0,1,2, \ldots$ We note that this resummation is the standard RG improvement of perturbative series, where large logarithms are summed up in the geometric series (or else a result of solution of RG equations). The crucial point is that most important physical thresholds are contained in $\Pi^{(0)}\left(Q^{2}\right)$ and have nothing to do with singularities of $\alpha_{B}\left(Q^{2}\right)$ (or of $\alpha_{s}\left(Q^{2}\right)$ ).

In a similar way the singularities which appear from the asymptotic solution of (46) for small $\alpha_{B}$, namely term $\ln \alpha_{B}\left(Q^{2}\right)$ does not produce additional logarithmic cuts and is a result of asymptotic expansion of Eq. (46), where in exact solution only meromorphic function $\alpha_{B}(s)$ is obtained.

Thus as we discussed above the equation (46) or its generalized form

$$
\alpha_{B}=\frac{4 \pi}{b_{0}\left[\ln \frac{m^{2}}{\Lambda^{2}}+\tilde{\psi}\left(Q^{2}\right)+\varphi\left(\frac{1}{\alpha_{B}}\right)\right]}
$$


where $\tilde{\psi}\left(Q^{2}\right)$ is given in (112) and $\varphi\left(\frac{1}{\alpha_{B}}\right)$ is given in (116), produce $\alpha_{B}\left(Q^{2}\right)$ which is meromorphic function of $Q^{2}$ in the whole complex plane of $Q^{2}$.

Let us now look more carefully at the creation of imaginary part of $\Pi\left(Q^{2}\right)$, which leads to the positive hadronic ratio $R(s)$. We start with $\Pi^{(0)}\left(Q^{2}\right)$ (85) and (83): $\Pi^{(0)}$ in SPT and BPT coincide asymptotically, namely

$$
\begin{gathered}
\Pi^{(0)}(S P T)=-\frac{e_{q}^{2} N_{c}}{12 \pi^{2}} \ln \frac{Q^{2}}{\mu^{2}} \\
\Pi^{(0)}(B P T)=-\frac{e_{q}^{2} N_{c}}{12 \pi^{2}} \ln \frac{Q^{2}+M_{0}^{2}}{\mu^{2}}
\end{gathered}
$$

Defining $s=-Q^{2}, \arg \left(M_{0}^{2}-s\right)=0$ for $s<M_{0}^{2}$, one has for large $s$

$$
\operatorname{Im} \Pi_{B P T}^{(0)}=\frac{\Pi^{(0)}(s+i \varepsilon)-\Pi^{(0)}(s-i \varepsilon)}{2 i}=\frac{e_{q}^{2} N_{c}}{12 \pi} \Theta\left(s-M_{0}^{2}\right)
$$

and for SPT one has on the r.h.s. $\Theta(s)$ instead of $\Theta\left(s-M_{0}^{2}\right)$.

Note however that the form (119) for $\Pi^{(0)}(\mathrm{BPT})$ is the asymptotics at large positive $Q^{2}$ and in getting the result (120) the analytic continuation of the logarithmic asymptotics was done unlawfully strictly speaking.

To calculate in BPT more rigorously, one should use (85) in the region $s>M_{0}^{2}$, and one gets

$$
\begin{gathered}
\operatorname{Im} \Pi^{(0)}(s+i \varepsilon)=-\frac{e_{q}^{2} N_{c} I m}{12 \pi^{2}} \psi\left(\frac{-s+M_{0}^{2}}{m^{2}}\right)=+\frac{e_{q}^{2} N_{c}}{12 \pi^{2}} \operatorname{Im} \sum_{k=0}^{\infty} \frac{m^{2}}{M_{0}^{2}-s+k m^{2}}= \\
\frac{e_{q}^{2} N_{c}}{12 \pi} m^{2} \sum_{k=0}^{\infty} \delta\left(M_{0}^{2}+k m^{2}-s\right)
\end{gathered}
$$

One can introduce the average of $\operatorname{Im} \Pi^{(0)}=(s+i \varepsilon)$ over some energy interval (this is the "duality interval" discussed e.g. in [7]) comprising $N$ poles

$$
\left\langle\operatorname{Im} \Pi^{(0)}\left(s_{0}+i \varepsilon\right)\right\rangle_{N}=\int_{s_{0}}^{s_{0}+N m^{2}} \frac{d s \operatorname{Im} \Pi^{(0)}(s+i \varepsilon)}{N m^{2}}=\frac{e_{q}^{2} N_{c}}{12 \pi}
$$

One can see that $(\sqrt{122})$ coincides with $(120)$ thus justifying the procedure of direct analytic continuation of the logarithms in (119), when the averaging over the duality interval is done.

One can also see that the standard SPT expression (119) yields the correct answer for the same reason: the imaginary part of asymptotics (both of SPT 
and BPT) coincides with the correct averaged imaginary part. We note that for SPT this check is impossible, since the correct procedure is not available.

We now turn to the more delicate point of analytic continuation of $\alpha(s)$. In BPT, as was discussed above, $\alpha_{B}(s)$ is given by $(118)$ or in "one-loop approximation" by (117) and is a meromorphic function, hence its analytic continuation is a direct and unique procedure. E.g. for $\alpha_{B}$ (117) separating one pole in $\psi\left(\frac{Q^{2}+M_{0}^{2}}{m^{2}}\right), M_{p}^{2}=M_{0}^{2}+n m^{2}$, one has in its vicinity

$$
\alpha_{B}\left(s \approx M_{n}^{2}+i \varepsilon\right)=\frac{4 \pi}{b_{0} \psi^{\prime}\left(z_{0}\right)\left(z-z_{0}\right)}
$$

where $z_{0}$ is to be found from the equation $\ln \frac{m^{2}}{\Lambda^{2}}+\psi\left(z_{0}\right)=0$, and $z=\frac{M_{0}^{2}-s}{m^{2}}$. (If one uses exact model expression (46) instead of "one-loop approximation" Eq. (117) the r.h.s. of (123) and subsequent (Eqs. (124) and (126) should be multiplied by the constant factor $\left(1-\frac{b_{0}}{4 \pi} \psi^{\prime}(\Delta)\right)$, which effectively decreases $\left.\operatorname{Im} \alpha_{B}\right)$. Using relations from Appendix, one gets

$$
\operatorname{Im} \alpha_{B}\left(s \cong M_{n}^{2}+i \varepsilon\right)=\frac{4 \pi^{2}}{b_{0}} \frac{m^{2} \delta\left(M_{n}^{2}-s-\delta_{0} m^{2}\right)}{\left[\frac{1}{\delta_{0}^{2}(n)}+S_{2}(n)+\zeta(2)\right]}
$$

where $\delta_{0}$ and $S_{k}(n)$ are defined in Appendix,

$$
\delta_{0}^{-1}(n)=\ln \frac{m^{2}}{\Lambda^{2}}-C+S_{1}(n)
$$

Let us now compare this exact procedure with the suggestive one, using the asymptotic form of (117), Eq.(111). As before, we have

$$
\operatorname{Im}\left(\bar{\alpha}_{B}\right) \equiv \frac{\bar{\alpha}_{B}(s+i \varepsilon)-\bar{\alpha}_{B}(s-i \varepsilon)}{2 i}=\frac{4 \pi^{2} \Theta\left(s-M_{0}^{2}\right)}{b_{0}\left\{\ln ^{2}\left|\frac{M_{0}^{2}-s}{\Lambda^{2}}\right|+\pi^{2}\right\}}
$$

One can notice on the other hand, that averaging over some duality interval in (124) yields

$$
\left\langle I m \alpha_{B}\right\rangle_{N m^{2}} \cong \frac{4 \pi^{2}}{b_{0}} \frac{1}{N} \sum_{k=0}^{N} \frac{1}{\delta^{-2}(n+k)+\frac{\pi^{2}}{3}},
$$

and this result as shown in Appendix asymptotically agrees with (125), specifically at large $s$ when the denominator in (125) grows. Now we turn to 
another definition of discontinuity od $\alpha_{B}$ which is widely used in $e^{+} e^{-}$annihilation and $\tau$ - decay. To make contact with SPT calculations consider instead of $\Pi\left(Q^{2}\right)$ the so called Adler function $D\left(Q^{2}\right)=\frac{Q^{2} d \Pi\left(Q^{2}\right)}{d Q^{2}}$ with the expansion

$$
D\left(Q^{2}\right)=\sum_{q} e_{q}^{2}\left\{1+\frac{\alpha_{s}\left(Q^{2}\right)}{\pi}+d_{2}\left(\frac{\alpha_{s}\left(Q^{2}\right)}{\pi}\right)^{2}+d_{3}\left(\frac{\alpha_{s}\left(Q^{2}\right)}{\pi}\right)^{3}+\ldots\right\}
$$

where coefficient $d_{3}$ was found in [21], and $d_{2}$ in [28]

$$
d_{2}(\overline{M S})=1.986-0.115 n_{f}, \quad \bar{d}_{3}(\overline{M S})=18.244-4.216 n_{f}+0.086 n_{f}^{2}
$$

The procedure suggested in [9] to go from $D\left(Q^{2}\right)$ to $R(s)$ is straightforward, provided one knows discontinuity of $\Pi\left(Q^{2}\right)$ (see discussion in [9], [8] and recent publication [29)

$$
R(s)=\frac{1}{2 \pi i} \int_{-s-i \varepsilon}^{-s+i \varepsilon} D(\sigma) \frac{d \sigma}{\sigma}
$$

where integration goes on both sides of the cut on real axis starting at $s=0$.

To proceed one inserts in (129) one - or two-loop expression for $\alpha_{s}\left(Q^{2}\right)$ and as the result one obtains an expansion for $R(s)$ [9]

$$
R(s)=\sum e_{q}^{2}\left\{1+\sum_{k=1} d_{k} \Phi\left\{\left(\frac{\alpha_{s}}{\pi}\right)^{k}\right\}\right\}
$$

where $\Phi\{\}$ is the transformation defined by (129). In this procedure the $\ln Q^{2}$ in the denominator of $\alpha_{s}\left(Q^{2}\right)$ transforms into $\ln s$ with coefficients less than unity and asymptotically tending to one.

Let us now consider what happens with our expression for $\alpha_{s}\left(Q^{2}\right)$ (117), which is valid not only for large $Q^{2}$ but in the whole $Q^{2}$ plane. We assume that in BPT the function $D_{B}\left(Q^{2}\right)$ is given by the same expansion (127), where only $\alpha_{s}$ should be replaced by $\alpha_{B}\left(Q^{2}\right)$ and we use (117) for $\alpha_{B}\left(Q^{2}\right)$ to insert into (129). In this way one obtains, keeping the notation of 9

$$
\Phi\left\{\left(\frac{\alpha_{B}}{\pi}\right)^{k}\right\}=\frac{1}{2 \pi i}\left(\frac{4}{b_{0}}\right)^{k} \sum_{n=0} \int_{s+i \varepsilon}^{s-i \varepsilon} \frac{d s^{\prime}}{\left[\psi^{\prime}\left(z_{0}\right)\left(z-z_{0}\right)\right]^{k} s^{\prime}} .
$$


As shown Appendix, $\psi^{\prime}\left(z_{0}\right) \approx\left(\ln \frac{M_{0}^{2}+n m^{2}}{\Lambda^{2}}\right)^{2}$. Here the pole of the $k$-th order is at $s^{\prime}=M_{0}^{2}+n m^{2}-\delta_{0}(n) m^{2}$, and $n$ satisfies condition $M_{0}^{2}+n m^{2} \leq s$. For arbitrary $k$ one obtains

$$
\Phi\left\{\left(\frac{\alpha_{B}}{\pi}\right)^{k}\right\}=0
$$

This is a property of the integral in (131) with the weight $\frac{d s}{s}$ that any function representable as a finite sum of poles yields zero since $\int_{s-i \varepsilon}^{s+i \varepsilon} \frac{d z}{z\left(z-s_{n}\right)^{k}}=0$. However in the asymptotic region of $s=\bar{s}(1+i \gamma), \quad \bar{s} \gg m^{2}$, when number of poles is large, one can use asymptotic form of $\alpha_{B},(112)$, and the result is

$$
\Phi\left\{\left(\frac{\alpha_{B}}{\pi}\right)\right\} \cong \frac{4}{b_{0}}\left(\frac{1}{\pi} \operatorname{arctg}\left(\frac{\pi}{\ln \frac{s}{\Lambda^{2}}}\right)+\frac{2\left(M_{0}\right)^{2}}{s\left(\ln \frac{s}{\Lambda^{2}}\right)^{3}}+\ldots\right) .
$$

This result can be most easily obtained from (129), where the contour of integration $C_{R}$ is modified to be a circle of radius $R=s$ with center at $\sigma=0$, namely

$$
R(s)=\frac{1}{2 i \pi} \int_{C_{R}} D(\sigma) \frac{d \sigma}{\sigma} ; \Phi\left\{\left(\frac{\alpha_{B}}{\pi}\right)^{k}\right\}=\frac{1}{2 i \pi} \int_{C_{R}} \frac{d \sigma}{\sigma}\left(\frac{\alpha_{B}(\sigma)}{\pi}\right)^{k}
$$

The result (132) is obtained from (134) trivially introducing angular variable $\sigma=s \exp (i \phi)$. For large $s, s \gg m^{2},\left(M_{0}\right)^{2}$ one can use in (134) asymptotic form of $\alpha_{B}(\sigma)$ from (111).

Thus one obtains for $R(s)$ :

$$
R(s)=N_{c} \sum_{q} e_{q}^{2}\left[1+\bar{\Phi}\left\{\frac{\alpha_{B}}{\pi}\right\}+d_{2} \bar{\Phi}\left\{\left(\frac{\alpha_{B}}{\pi}\right)^{2}\right\}+d_{3} \bar{\Phi}\left\{\left(\frac{\alpha_{B}}{\pi}\right)^{3}\right\}+\ldots\right]
$$

Comparing with $\Phi\left\{\left(\frac{\alpha_{s}}{\pi}\right)^{k}\right\}$ from Radyushkin [9], one can see very close correspondence with our $\bar{\Phi}\left\{\left(\frac{\alpha_{B}}{\pi}\right)^{k}\right\}$.

\section{Discussion and Conclusions}

We have developed in this paper the background perturbation theory (BPT) in the large $N_{c}$ limit, when all physical amplitudes and their perturbative expansions have only isolated singularities (poles). 
Using this fact, we have proposed a nonperturbative solution to $\beta(\alpha)$ and $\alpha\left(Q^{2}\right)$ containing all terms of loop expansion.

By choosing a specific meromorphic function we have modelled both $\beta\left(\alpha_{B}\right)$ and $\alpha_{B}\left(Q^{2}\right)$ satisfying necessary criteria of analyticity of $\alpha_{B}$ in the Euclidean region of $Q^{2}$, and analyticity of $\beta(\alpha)$ for positive values of $\alpha_{B}$.

We have constructed the time-dependent old-fashioned perturbation theory to investigate analytic properties of different terms of perturbation series, and have shown that those reduce to isolated poles (of first and higher degree) shifted with respect to unperturbed bound state positions.

This enables us to modify original ansatz for $\beta(\alpha)$ and $\alpha_{B}\left(Q^{2}\right)$ to achieve a full correspondence with standard perturbative expansion of $\beta(\alpha)$.

Finally, we have studied the problem of analyticity of $\alpha_{B}\left(Q^{2}\right)$ and of analytic continuation into region of time-like $Q^{2}$, where $\alpha_{B}\left(Q^{2}\right)$ has singularities, and have compared this procedure with analytic continuation in SPT.

We have found a striking similarity in the perturbative series for $R(s)$ in case of SPT and BPT at large $s$. However our BPT series has correct physical singularities at large $N_{c}$ in contrast to SPT.

More detailed calculations and comparison with experiment may decide on the applicability of both methods and are planned for the subsequent publication.

Financial support of the RFFI grant 00-02-17836 and INTAS grants 00110 and 00366 is gratefully acknowledged. 


\section{Appendix}

To find poles of $\alpha_{B}(s)=\frac{4 \pi}{b_{0}\left[\ln \frac{m^{2}}{\Lambda^{2}}+\psi\left(\frac{M_{0}^{2}-s}{m^{2}}\right)\right]}$ one must find the roots $z_{0}(n)$ of the equation

$$
\ln \frac{m^{2}}{\Lambda^{2}}+\psi\left(z_{0}\right)=0
$$

which can be represented as

$$
z_{0}(n)=-n+\delta, \quad \delta \ll 1, \quad n=0,1,2 .
$$

The following relations will be useful

$$
\begin{gathered}
\psi(-n+\delta)=\sum_{k=0}^{n} \frac{1}{k-\delta}+\psi(1+\delta), \quad n \geq 0, \\
\psi(1+\delta)=-C+\delta\{\zeta(2)-\delta \zeta(3)+\ldots\}
\end{gathered}
$$

where $\zeta(2)=\frac{\pi^{2}}{6}, \quad \zeta(3)=1.202$.

Hence one can write an expansion in powers of $\delta$

$$
\psi(-n+\delta)=-\frac{1}{\delta}-C+S_{1}(n)+\delta\left(1+S_{2}(n)+\zeta(2)\right)+O\left(\delta^{2}\right)
$$

where $S_{k}(n)=\sum_{k=1}^{n} \frac{1}{k}, \quad C=0.577$, and Eq.(A.1) takes the form

$$
-\frac{1}{\delta}-C+S_{1}(n)+\delta\left(1+S_{2}(n)+\zeta(2)\right)=-\ln \frac{m^{2}}{\Lambda^{2}} .
$$

To the lowest order one has

$$
\delta \cong \delta_{0}=\frac{1}{\ln \frac{m^{2}}{\Lambda^{2}}-C+S_{1}(n)}+O\left(\delta_{0}^{3}\right) .
$$

In a similar way one obtains $\psi^{\prime}\left(z_{0}\right)$,

$$
\psi^{\prime}\left(z_{0}\right)=\frac{1}{\delta^{2}}+S_{2}(n)+\zeta(2)+O(\delta) .
$$

For $n \gg 1, S_{2}(n) \approx \zeta(2)=\frac{\pi^{2}}{6}$. 
In this way $\alpha_{B}$ in the vicinity of the pole is

$$
\alpha_{B}\left(s \approx M_{n}^{2}+i \varepsilon\right)=\frac{4 \pi}{b_{0}\left[\delta_{0}^{-2}(n)+S_{2}(n)+\zeta(2)\right]\left(M_{n}^{2}-\delta_{0} m^{2}-s\right)}
$$

where $M_{n}^{2}=M_{0}^{2}+n m^{2}$.

Now for $S_{1}(n)$ the following asymptotics holds true

$$
S_{1}(n)=C+\ln n+\frac{1}{2 n}+O\left(\frac{1}{n^{2}}\right) .
$$

Therefore $\delta_{0}$ (A.7) can be rewritten as

$$
\delta_{0} \cong \frac{1}{\ln \frac{m^{2}}{\Lambda^{2}}+\ln \left(n+\frac{1}{2}\right)}=\frac{1}{\ln \frac{m^{2}\left(n+\frac{1}{2}\right)}{\Lambda^{2}}} \cong \frac{1}{\ln \frac{M_{n}^{2}}{\Lambda^{2}}}
$$

and the averaged imaginary part of $\alpha_{B}$ in (126) has the form

$$
\left\langle I m \alpha_{B}\right\rangle_{N m^{2}}=\frac{4 \pi^{2}}{b_{0} N} \sum_{k=0}^{n} \frac{1}{\left(\ln \frac{M_{n+k}^{2}}{\Lambda^{2}}\right)^{2}+\frac{\pi^{2}}{3}} \approx \frac{4 \pi^{2}}{b_{0}\left(\ln \frac{\bar{M}^{2}}{\Lambda^{2}}\right)^{2}}
$$

where $\bar{M}^{2} \sim s$ is in the middle of the averaging interval, which is asymptotically close to the expression in Eq. (125).

\section{References}

[1] F.J.Yndurain, The Theory of Quark and Gluon Interactions, 3d edition, Springer-Verlag, Berlin-Heidelberg, 1999.

[2] L.D.Faddeev and A.A.Slavnov, Gauge Fields, Benjamin, New York, 1980

[3] See e.g. discussion in chapter 10 of [1]

[4] A.M.Badalian, D.S.Kuzmenko, hep-ph/0104097

[5] G.'t Hooft, In The Whys of Subnuclear Physics, Proc. Int. School, Erice, 1977 (A.Zichichi, ed) Plenum, New York

[6] M.A.Shifman, A.I.Vainshtein, V.I.Zakharov, Nucl. Phys. B147 385 (1979) 
[7] M.A.Shifman (ed) Vacuum Structure and QCD Sum Rules, NorthHolland, 1992

S.Narison, QCD Spectral sum Rules, World Scientific, 1989

[8] D.V.Shirkov, hep-ph/0012283, hep-ph/0107282

[9] A.Radyushkin, Dubna JINR preprint E-2-82-159 (1982), and hepph/9907228;

N.V.Krasnikov, A.A.Pivovarov, Phys. Lett. 116B 168 (1982)

[10] G.'tHooft, Nucl. Phys. B72 461 (1974)

[11] B.S.De Witt, Phys. Rev. 1621195, 1239 (1967) ;

J.Honerkamp, Nucl. Phys. B48 269 (1972);

G.'t Hooft, Nucl. Phys. B62 44 (1973), Lectures at Karpacz, in: Acta Univ. Wratislaviensis 368345 (1976) ;

L.F.Abbot Nucl. Phys. 185189 (1981).

[12] Yu.A.Simonov, Yad. Fiz. 58113 (1995), hep/ph/9311247, JETP Letters 57513 (1993)

[13] Yu.A.Simonov, In: Lecture Notes in Physics, v.479 p.139 (1996) , Springer-Verlag, Berlin-Heidelberg

[14] A.M.Badalian and Yu.A.Simonov, Phys. At. Nucl. 60630 (1997)

[15] A.M.Badalian and V.L.Morgunov, Phys. Rev. D60 116008 (1999), hepph/9901430

[16] A.M.Badalian and B.L.G.Bakker, Phys. Rev. D62 094031 (2000), hepph/0004021

[17] Yu.A.Simonov, hep-ph/9712250

[18] H.G.Dosch and Yu.A.Simonov, Phys. Lett. B205 339 (1988)

[19] M.Campostrini, A.Di Giacomo, G.Mussardo, z.Phys. C25 173 (1984);

A.Di Giacomo and H.Panagopoulos, Phys. Lett. B285 133 (1992) ;

A.Di Giacomo, E.Meggiolaro and H.Panagopoulos, Nucl. Phys. B483 371 (1997), hep-lat/9603017 
[20] G.Sterman, in Lecture Notes in Physics, v. 479, p. 209, Springer-Verlag, Berlin-Heidelberg, 1996.

[21] S.G.Gorishny, A.L.Kataev and S.A.Larin, Phys. Lett. B259 144 (1991); M.A.Samuel and L.R.Surguladze, Phys. Rev. Lett. 66560 (1991)

[22] A.Yu. Dubin, A.B.Kaidalov, Yu.A.Simonov, Yad. Fiz. 56213 (1993); Phys. Lett. 32341 (1994)

[23] Yu.A.Simonov; Nonperturbative corrections to the quark self-energy, hep-ph/0105141, Phys. Lett. B (in press).

[24] P.Cea, G.Nardulli and G.Preparata, Z. Phys. C16 135 (1982); Phys. Lett. B115 310 (1982)

[25] Yu.A.Simonov, JETP Lett. 69505 (1999); Phys. Rep. 320265 (1999)

[26] K.G.Chetyrkin, S.Narrison and V.I.Zakharov, hep-ph/9811275

[27] V.S.Popov, V.M.Vainberg, V.D.Mur, JETP Lett. 41539 (1985), Sov. J.Nucl. Phys. 44, 714 (1986);

B.M.Karnakov, V.D.Mur, V.S.Popov, V.M.Vainberg, V.D.Mur, JETP Lett. 41535 (1985), Sov. J. Nucl. Phys. 44, 714 (1986);

B.M.Karnakov, V.D.Mur, V.S.Popov JETP 80, 360 (1995)

[28] K.G.Chetyrkin, A.L.Kataev, F.V.Tkachov, Nucl. Phys.B174 345 (1980);

M.Dine, J.Sapirstein, Phys. rev. Lett 43668 (1979) ;

W.Celmaster, R.Gonsalvez, Phys. ReV. D21 3112 (1980)

[29] D.V.Shirkov, hep-ph/0105317

[30] Review of Particle Physics, Europ. Phys. Journ. C15 229 (2000) 